0362-546X(94)00136-7

\title{
ON A NONLOCAL ZAKHAROV EQUATION
}

\author{
B. BIDÉGARAY \\ Centre de Mathématiques et Leurs Applications, ENS de Cachan, 61 avenue du président Wilson, \\ 94235 Cachan CEDEX, France
}

(Received 31 July 1993; received for publication 4 July 1994)

Key words and phrases: Cauchy problem, nonlocal Zakharov equation, functional space.

\section{INTRODUCTION}

Our aim is to prove some results about a nonlocal Zakharov equation introduced by Zakharov (see $[1,2]$ ). The derivation of this system is carried out for $x \in \mathbb{R}^{3}$, however, we will suppose that $x \in \mathbb{R}^{N}, N=1,2,3$.

We study the following system

$$
\left\{\begin{array}{l}
i \dot{\phi}+\Delta \phi=-B(n \phi), \\
\lambda^{-2} \ddot{n}-\Delta n=\Delta|\phi|^{2},
\end{array}\right.
$$

where $B=\nabla \Delta^{-1} \nabla$.

We consider the initial value problem, that is

$$
\left\{\begin{array}{l}
\phi(x, 0)=\phi_{0}(x), \\
n(x, 0)=n_{0}(x), \\
n_{t}(x, 0)=n_{1}(x) .
\end{array}\right.
$$

The article is divided into four sections. In Section 2, we derive the equation from the physical equations according to Zakharov (see [1]). In Section 3, we study the local Cauchy problem for $\left(\phi_{0}, n_{0}, n_{1}\right) \in H^{2} \times H^{1} \times L^{2}$. In Section 4, we study the limit of the solution to the equation when $\lambda$ tends to zero.

The nonlocal Zakharov equation may be connected with two other equations: the classical Zakharov equation and a nonlinear nonlocal Schrödinger equation.

The classical Zakharov equation is the same one with $B=-I$. There is a link between the two problems. For example, they are the same in a one-dimensional space and also if we only consider radial solutions (because $-B$ is the $L^{2}$-projection on the gradients (see [3]). The point is to try to adapt the results about the classical equation. We may cite different people who worked on this equation: Added and Added [4, 5], Sulem and Sulem [6], Schochet and Weinstein [7], and Ozawa and Tsutsumi [8].

For the existence and the uniqueness for the Cauchy problem, we reason as in [8]. It is possible to adapt the method because of the continuity of the nonlocal operator $B$ in a large number of spaces. The same results on the Cauchy problem would be obtained if we have another operator instead of $B$, provided we always have these continuity results.

To pass to the limit when $\lambda$ tends to $\infty$, we adapt the proof in [7]. Lots of complications are due to the nonlocal term. First of all we have to write the initial system as the dispersive 
perturbation of a symmetric hyperbolic system, the nonlocal term yields some additional terms which make the derivation far more complicated. We then encounter some difficulties in estimating the different nonlocal operators of this new system and need the use of the theory of commutators to solve these new problems. As for the previous problem, we may want to extend our results to some other operator $B$. We need the same properties as before, and some others. For example, we need to be able to define an operator $A$ such that $\nabla B \Phi=A \nabla \Phi$.

A nonlinear, nonlocal Schrödinger equation is studied by Colin [3, 9]. It consists in taking $\lambda=\infty$. He obtains some results about the local and global Cauchy problem, some finite time blow-up results and also standing waves and their stability. The limit we obtain when $\lambda$ tends to $\infty$ turns out to be the solutions to this equation.

\section{ORIGIN OF THE NONLOCAL ZAKHAROV SYSTEM}

This article deals with some equations introduced by Zakharov (cf. e.g. [1, 2]), to describe Langmuir plasma turbulence. The physical description follows exactly these two articles.

We consider the hydrodynamical equations, the system of the Vlasov equations for the particle distribution functions and the Maxwell equations for the fields. This system is quite complicated, so we first simplify it. The idea is based on the fact that we can distinguish slow and fast processes in a plasma. We assume

- the plasma is sufficiently uniform,

- the magnetic field is sufficiently weak,

- the nonlinearity level is not too high,

- there are no transverse high-frequency electromagnetic waves,

then the fastest process is the Langmuir oscillation, whose period is $\tau_{L} \sim 1 / \omega_{\mathrm{pl}}, \omega_{\mathrm{pl}}$ being the Langmuir frequency.

The other time scale (when there is no magnetic field) is the period of ion-acoustical oscillations, their minimal value being $\sqrt{m_{i} / m_{e}}$ times higher than $\tau_{L}$, where $m_{i}$ and $m_{e}$ denote the ion and electron masses.

We average the dynamical equations on a period $\tau_{L}$. We only consider long wave oscillations with phase velocities far larger than thermal ones. We neglect quasi-linear effects.

We also neglect interactions between the different high-frequency oscillations, and obtain the linearized hydrodynamical equations

$$
\begin{gathered}
\frac{\partial}{\partial t} \delta n_{e}+\operatorname{div}\left(n_{0}+\delta n\right) \mathbf{V}_{e}=0 \\
\frac{\partial}{\partial t} \delta \mathbf{V}_{e}+\frac{3 V_{T_{e}}^{2}}{n} \nabla \delta n=\frac{\mathrm{e}}{m_{e}} \mathbf{E}
\end{gathered}
$$

Maxwell's equation reads

$$
\frac{1}{\mathrm{c}^{2}} \frac{\partial^{2} \mathbf{E}}{\partial t^{2}}+\text { curl curl } \mathbf{E}+\frac{4 \pi \mathrm{e}}{\mathrm{c}^{2}}\left(n_{0}+\delta n\right) \frac{\partial}{\partial t} \delta \mathbf{V}_{e}=0
$$

Moreover, we suppose that

$$
\begin{gathered}
n=n_{0}+\delta n+\delta n_{e}, \\
\delta n, \delta n_{e} \ll n_{0},
\end{gathered}
$$


where $n$ is the electron density, $\delta n$ is the given low frequency plasma nonuniformity and $\delta n_{e}$ is the density variation due to the Langmuir oscillations. $\mathbf{E}$ is the electric field and $\delta \mathbf{V}_{e}$ is the variation of the electron velocity. $\mathrm{c}$ is the ion sound velocity. Thus, we have

$$
\frac{1}{c^{2}}\left(\frac{\partial^{2} \mathbf{E}}{\partial t^{2}}+\omega_{\mathrm{pl}}^{2} \mathbf{E}\right)+\operatorname{curl} \operatorname{curl} \mathbf{E}-\frac{3 V_{T_{e}}^{2}}{\mathrm{c}^{2}} \nabla \operatorname{div} \mathbf{E}+\omega_{\mathrm{pl}}^{2} \frac{\delta n}{\mathrm{c}^{2} n_{0}} \mathbf{E}=0 .
$$

Next, we suppose that $\mathbf{E}=\frac{1}{2}\left(\tilde{\mathbf{E}} \exp \left(-i \omega_{\mathrm{pl}} t\right)+\tilde{\mathbf{E}}^{*} \exp \left(i \omega_{\mathrm{p} 1} t\right)\right)$, where $\tilde{\mathbf{E}}$ slowly varies $\left(\partial \tilde{E} / \partial t \ll \omega_{\mathrm{pl}} \tilde{E}\right)$. Neglecting $\partial^{2} \tilde{E} / \partial t^{2}$, we have

$$
\frac{\partial^{2} \mathbf{E}}{\partial t^{2}}=-\omega_{\mathrm{pl}}^{2} \mathbf{E}+i \omega_{\mathrm{pl}}\left(\frac{\partial \tilde{\mathbf{E}}}{\partial t} \exp \left(-i \omega_{\mathrm{pl}} t\right)+\frac{\partial \tilde{\mathbf{E}}^{*}}{\partial t} \exp \left(i \omega_{\mathrm{pl}} t\right)\right)
$$

Substituting this in (4), we obtain

$$
-2 i \frac{\omega_{\mathrm{p} 1}}{\mathrm{c}^{2}} \frac{\partial \tilde{\mathbf{E}}}{\partial t}+\operatorname{curl} \operatorname{curl} \tilde{\mathbf{E}}-\frac{3 V_{T_{e}}^{2}}{\mathrm{c}^{2}} \nabla \operatorname{div} \tilde{\mathbf{E}}=-\omega_{\mathrm{pl}}^{2} \frac{\delta n}{\mathrm{c}^{2} n_{0}} \tilde{\mathbf{E}} .
$$

We need a second equation in order to relate $\delta n$ and $|\tilde{E}|^{2}$.

We suppose that the electrons are distributed according to the Boltzmann law

$$
n=n_{0} \exp \left\{-\frac{\left(\mathrm{e} \varphi_{\mathrm{el}}-\phi\right)}{T_{e}}\right\}, \quad \frac{\delta n}{n_{0}}=\frac{\mathrm{e} \varphi_{\mathrm{el}}-\phi}{T_{e}} \ll 1 .
$$

The ion distribution function obeys Vlasov's equation

$$
\frac{\partial f_{i}}{\partial t}+(V \cdot \nabla) f_{i}-\frac{\mathrm{e}}{m_{i}} \nabla \varphi_{\mathrm{el}} \frac{\partial f_{i}}{\partial V}=0, \quad \text { and } \quad \delta n_{i}=\frac{\mathrm{e} n_{0}}{T_{e}}\left(\varphi_{\mathrm{el}}-\phi\right)
$$

We set $\tilde{\mathbf{E}}=\boldsymbol{\nabla} \psi$, so $\operatorname{div} \tilde{\mathbf{E}}=\Delta \psi$,

$$
\begin{gathered}
-2 i \frac{\omega_{\mathrm{pl}}}{\mathrm{c}^{2}} \frac{\partial}{\partial t}(\operatorname{div} \tilde{\mathbf{E}})-\frac{3 V_{T_{e}}^{2}}{\mathrm{c}^{2}} \operatorname{div} \nabla \operatorname{div} \tilde{\mathbf{E}}=-\frac{\omega_{\mathrm{pl}}^{2}}{\mathrm{c}^{2}} \operatorname{div}\left(\frac{\delta n}{n_{0}} \tilde{\mathbf{E}}\right), \\
-2 i \frac{\omega_{\mathrm{pl}}}{\mathrm{c}^{2}} \frac{\partial}{\partial t} \Delta \psi-\frac{3 V_{T_{e}}^{2}}{\mathrm{c}^{2}} \Delta^{2} \psi=-\frac{\omega_{\mathrm{pl}}^{2}}{\mathrm{c}^{2}} \operatorname{div}\left(\frac{\delta n}{n_{0}} \nabla \psi\right),
\end{gathered}
$$

thus

$$
\Delta\left(2 i \omega_{\mathrm{pl}} \psi_{\imath}+3 V_{T_{e}}^{2} \Delta \psi\right)=\omega_{\mathrm{pl}}^{2} \operatorname{div}\left(\frac{\delta n}{n_{0}} \nabla \psi\right)
$$

We multiply (7) by $\psi^{*}$ and integrate by parts. The imaginary part yields that

$$
N_{0}=\int|\nabla \psi|^{2} \mathrm{~d} x
$$

is conserved. 
First hypothesis. The nonlinear phenomena have such a long period $\left(\tau^{-1} \ll \mathrm{k} V_{T_{i}}\right)$ that the ions have enough time to reach the Boltzmann distribution law in a low-frequency electric field

$$
\frac{\delta n}{n_{0}}=-\mathrm{e} \frac{\varphi_{\mathrm{el}}}{T_{i}}
$$

Now, we also know that $\delta n / n_{0}=\left(\mathrm{e} \varphi_{\mathrm{el}}-\phi\right) / T_{e}$, therefore,

$$
\frac{\delta n}{n_{0}}=\frac{-\phi}{T_{i}+T_{e}} .
$$

$\phi$ equals $\left(\mathrm{e}^{2} / 4 m_{e} \omega_{\mathrm{pl}}^{2}\right)|\tilde{\mathbf{E}}|^{2}$, hence

$$
\frac{\delta n}{n_{0}}=\frac{-\mathrm{e}^{2}}{4 m_{e} \omega_{\mathrm{pl}}^{2}} \frac{|\nabla \psi|^{2}}{\left(T_{i}+T_{e}\right)}=\frac{-\varepsilon_{0}}{4 n_{0}} \frac{|\nabla \psi|^{2}}{\left(T_{i}+T_{e}\right)} .
$$

This result together with (7) yields

$$
\begin{aligned}
\Delta\left(2 i \omega_{\mathrm{pl}} \psi_{t}+3 V_{T_{e}}^{2} \Delta \psi\right) & =\frac{-\mathrm{e}^{2}}{4 m_{e}\left(T_{i}+T_{e}\right)} \operatorname{div}\left(|\nabla \psi|^{2} \nabla \psi\right), \\
\Delta\left(i \psi_{t}+\frac{3}{2} \frac{V_{T_{e}}^{2}}{\omega_{\mathrm{pl}}} \Delta \psi\right) & =\frac{-\mathrm{e}^{2}}{4 m_{e} \omega_{\mathrm{pl}}\left(T_{i}+T_{e}\right)} \operatorname{div}\left(|\nabla \psi|^{2} \nabla \psi\right), \\
\Delta\left(i \psi_{t}+\frac{3}{2} \lambda_{D} \omega_{\mathrm{pl}} \Delta \psi\right) & =\frac{-\varepsilon_{0} \omega_{\mathrm{pl}}}{8 n_{0}\left(T_{i}+T_{e}\right)} \operatorname{div}\left(|\nabla \psi|^{2} \nabla \psi\right) .
\end{aligned}
$$

Second hypothesis. $\tau^{-1} \gg \mathrm{k} V_{T_{i}}$. For low-frequency motions the hydrodynamical approximation is valid

$$
\left(\frac{\partial^{2}}{\partial t^{2}}+2 \gamma_{s} \frac{\partial}{\partial t}-c_{s}^{2} \Delta\right) \delta n=\frac{1}{16 \pi m_{i}} \Delta|E|^{2}
$$

We assume that the damping rate $\gamma_{s}$ is zero.

After some changes of scale, we obtain the equations, we study. For the first hypothesis, we obtain

$$
\Delta\left(i \psi_{t}+\Delta \psi\right)=\operatorname{div}\left(|\nabla \psi|^{2} \nabla \psi\right) .
$$

We can find some results concerning this equation in $[3,9]$. For the second hypothesis, we have the following system

$$
\left\{\begin{array}{l}
\Delta\left(i \psi_{t}+\Delta \psi\right)=\operatorname{div}(n \nabla \psi), \\
\frac{1}{\mathrm{c}^{2}} n_{t t}-\Delta n=\Delta\left(|\nabla \psi|^{2}\right) .
\end{array}\right.
$$




\section{EXISTENCE AND UNIQUENESS FOR THE CAUCHY PROBLEM}

In the study of the Cauchy problem, we will omit the constant $\mathrm{c}^{2}$. It is obvious to see that the result will be valid for every value of $c$. Thus we study

$$
\left\{\begin{array}{l}
\Delta(i \psi+\Delta \psi)=\nabla \cdot(n \nabla \psi) \\
\ddot{n}-\Delta n=\Delta|\nabla \psi|^{2}
\end{array}\right.
$$

that is

$$
\begin{gathered}
\left\{\begin{array}{l}
i \dot{\psi}+\Delta \psi=\Delta^{-1} \nabla \cdot(n \nabla \psi), \\
\ddot{n}-\Delta n=\Delta|\nabla \psi|^{2},
\end{array}\right. \\
\left\{\begin{array}{l}
i \nabla \dot{\psi}+\Delta \nabla \psi=\nabla \Delta^{-1} \nabla \cdot(n \nabla \psi), \\
\ddot{n}-\Delta n=\Delta|\nabla \psi|^{2} .
\end{array}\right.
\end{gathered}
$$

We set $\phi=\nabla \psi$. This leads to the system that we are going to study

$$
\left\{\begin{array}{l}
i \dot{\phi}+\Delta \phi=\nabla \Delta^{-1} \nabla \cdot(n \phi) \\
\ddot{n}-\Delta n=\Delta|\phi|^{2}
\end{array}\right.
$$

In the study, we omit the fact that $\phi$ is a gradient. We nevertheless have $i \dot{\phi}+\Delta \phi=\nabla f$ with $f=\Delta^{-1} \nabla \cdot(n \phi)$. Taking the Fourier transform, we get

$$
\begin{aligned}
i \widehat{\phi_{t}}-|\xi|^{2} \hat{\phi} & =i \xi \hat{f} \\
\widehat{\phi_{t}}+i|\xi|^{2} \hat{\phi} & =\xi \hat{f} \\
\frac{\mathrm{d}}{\mathrm{d} t}\left(\hat{\phi} \mathrm{e}^{i|\xi|^{2} t}\right) & =\xi \hat{f} \mathrm{e}^{i|\xi|^{2} t} \\
\hat{\phi} \mathrm{e}^{i|\xi|^{2} t}-\hat{\phi}(0) & =\xi \int_{0}^{t} \hat{f}(\xi, s) \mathrm{e}^{i|\xi|^{2} s} \mathrm{~d} s
\end{aligned}
$$

As we have $\hat{\phi}(0)=\xi \hat{\psi}(0)$,

$$
\bar{\phi}(\xi, t)=\xi\left(\bar{\phi}(\xi, 0) \mathrm{e}^{-i|\xi|^{2} t}+\int_{0}^{t} \hat{f}(\xi, s) \mathrm{e}^{-i|\xi|^{2}(t-s)} \mathrm{d} s\right),
$$

and $\phi$ is a gradient.

These computations are quite justified. We set $B=\nabla(-\Delta)^{-1} \nabla$. This operator is homogeneous of order 0 in the Fourier variable. Calderón-Zygmund's theorem tells us that $B$ is continuous in $L^{p}\left(\mathbb{R}^{m}\right)$, for all $1<p<\infty$, i.e.

$$
\exists C_{p} / \forall f \in L^{p}\|B f\|_{L^{p}} \leq C_{p}\|f\|_{L^{p}} .
$$

A simple argument tells us that $B$ is also continuous in every $W^{s, p}$, for all $1<p<\infty$

$$
\exists C_{s, p} / \forall f \in W^{s, p}\|B f\|_{W^{s, p}} \leq C_{s, p}\|f\|_{W^{s, p}} .
$$


We now follow the work of Ozawa and Tsutsumi [8]. The system (12) reads

$$
\left\{\begin{array}{l}
\ddot{i \phi}+\Delta \phi=-B(n \phi), \\
\ddot{n}-\Delta n=\Delta|\phi|^{2} .
\end{array}\right.
$$

Setting $F=\partial_{t} \phi$, we formally get

$$
\left\{\begin{array}{l}
i \dot{F}+\Delta F=-B\left(\partial_{t} n\left(\phi_{0}+\int_{0}^{t} F \mathrm{~d} s\right)+n F\right) \\
\ddot{n}-\Delta n=\Delta|\phi|^{2} \\
\phi=(-\Delta+1)^{-1}\left\{i F+B\left(n\left(\phi_{0}+\int_{0}^{t} F \mathrm{~d} s\right)\right)+\left(\phi_{0}+\int_{0}^{t} F \mathrm{~d} s\right)\right\} .
\end{array}\right.
$$

We consider the following initial conditions

$$
\left\{\begin{array}{l}
F(0)=i\left(\Delta \phi_{0}+B\left(n_{0} \phi_{0}\right)\right)=F_{0} \\
n(0)=n_{0} \\
\partial_{t} n(0)=n_{1} .
\end{array}\right.
$$

We work in $\mathbb{R}^{N}$ with $1 \leq N \leq 3$.

We set

$$
X=\left[L^{\infty}\left(I ; L^{2}\right) \cap L^{8 / N}\left(I ; L^{4}\right)\right] \oplus\left[L^{\infty}\left(I ; H^{1}\right) \cap W^{1, \infty}\left(I ; L^{2}\right)\right],
$$

where $I=[0, T]$. We suppose that $\left(\phi_{0}, n_{0}, n_{1}\right) \in H^{2} \times H^{1} \times L^{2}$, and we set

$$
a=\max \left\{\left\|\phi_{0}\right\|_{L^{2}},\left\|\phi_{0}\right\|_{L^{4}},\left\|\Delta \phi_{0}+B\left(n_{0} \phi_{0}\right)\right\|_{L^{2}},\left\|n_{0}\right\|_{H^{1}}+\left\|n_{1}\right\|_{L^{2}}\right\}
$$

(as we have $\left(\phi_{0}, n_{0}, n_{1}\right) \in H^{2} \times H^{1} \times L^{2}, a$ is finite).

To begin with, we want to obtain the existence and the uniqueness of a solution of (16)-(17) in $X$ using a fixed point method. We set $N=\left(N_{1}, N_{2}\right)$ with

$$
\left\{\begin{array}{l}
N_{1}[F, n](t)=U(t) F_{0}+i \int_{0}^{t} U(t-s)\left\{B\left(\partial_{s} n\left(\phi_{0}+\int_{0}^{s} F \mathrm{~d} \tau\right)+n F\right)\right\} \mathrm{d} s \\
N_{2}[F, n](t)=\cos (\omega t) n_{0}+\omega^{-1} \sin (\omega t) n_{1}+\int_{0}^{t} \omega^{-1} \sin (\omega(t-s)) \Delta|\phi(s)|^{2} \mathrm{~d} s \\
(-\Delta+1) \phi=\left\{i F+B\left(n\left(\phi_{0}+\int_{0}^{t} F \mathrm{~d} s\right)\right)+\left(\phi_{0}+\int_{0}^{t} F \mathrm{~d} s\right)\right\}
\end{array}\right.
$$

where $U(t)$ is the semigroup generated by the Schrödinger operator and $\omega$ denotes $\sqrt{-\Delta}(|\xi|$ in Fourier variable).

We set

$$
\begin{gathered}
Y=\left\{(F(t), n(t)) \in X /\|F\|_{L^{\infty}\left(I ; L^{2}\right)} \leq 2 a,\|F\|_{L^{8 / N}\left(I ; L^{4}\right)} \leq 2 \delta a,\right. \\
\left.\|n\|_{L^{\infty}\left(I ; H^{1}\right)} \leq 2 a,\left\|\frac{\mathrm{d} n}{\mathrm{~d} t}\right\|_{L^{\infty}\left(I ; L^{2}\right)} \leq 2 a\right\} .
\end{gathered}
$$

We first show that $N$ is a contraction from $Y$ into $Y$. To this end we use the following lemmas. 
LEMMA 1. $L^{p}-L^{q}$ estimate (see $\left.[10,11]\right)$. If $2 \leq p \leq_{\infty}$ and $1 / p+1 / q=1$, then $\|U(t) v\|_{L^{p}} \leq$ $(4 \pi|t|)^{N / 2+N / p}\|v\|_{L^{q}}, t \neq 0$.

Lemma 2. Strichartz estimate (see [12]). We set $\alpha(N)=\infty$ if $N=1,2 ; \alpha(N)=2 N /(N-2)$ if $N \geq 3$. If $2 \leq q<\alpha(N)$ and $(N / 2-N / q) r=2$, then there exists $K_{1}(N, q)$ such that $\|U(\cdot) v\|_{L^{r}\left(\mathbb{R} ; L^{q}\right)} \leq K_{1}\|v\|_{L^{2}}$ for all $v \in L^{2}$.

LEMmA 3. (See [13].) If $1 \leq q^{\prime}, r^{\prime} \leq 2,2 \leq q<\alpha(N)$ and $(N / 2-N / q) r=2$, then there exists $K_{2}\left(N, q^{\prime}\right)$ such that $\left\|\int_{0}^{t} U(t-s) f(s) \mathrm{d} s\right\|_{L^{\infty}\left(I ; L^{2}\right)} \leq K_{2}\|f\|_{L^{r^{\prime}}\left(I ; L^{q^{\prime}}\right)} \forall f \in L^{r^{\prime}}\left(I ; L^{q^{\prime}}\right)$.

LEMMA 4. For all $t \in \mathbb{R}^{+}$, for all $v \in L^{2}$, we have

$$
\|\cos (\omega t) v\|_{L^{2}} \leq\|v\|_{L^{2}}, \quad\left\|\left(1+\omega^{2}\right)^{1 / 2} \omega^{-1} \sin (\omega t) v\right\|_{L^{2}} \leq(1+t)\|v\|_{L^{2}}
$$

First step. $N$ maps $Y$ into $Y$. The lemma 2 yields that

$$
\left\|U(\cdot) i\left(\Delta \phi_{0}+B\left(n_{0} \phi_{0}\right)\right)\right\|_{L^{8 / N}\left(\mathbb{R} ; L^{4}\right)} \leq K_{1}\left\|\Delta \phi_{0}+B\left(n_{0} \phi_{0}\right)\right\|_{L^{2}} \leq \delta a .
$$

This fixes $\delta$. We also know that

$$
\left\|U(\cdot) i\left(\Delta \phi_{0}+B\left(n_{0} \phi_{0}\right)\right)\right\|_{L^{\infty}\left(\mathbb{R} ; L^{2}\right)}=\left\|\Delta \phi_{0}+B\left(n_{0} \phi_{0}\right)\right\|_{L^{2}} \leq a,
$$

because $U(t)$ is unitary on $L^{2}$. Using the first lemma and the continuity property (13) of $B$, we have

$$
\begin{aligned}
& \left\|N_{1}[F, n](t)\right\|_{L^{4}} \\
& \leq\left\|U(t) i\left(\Delta \phi_{0}+B\left(n_{0} \phi_{0}\right)\right)\right\|_{L^{4}}+\int_{0}^{t}\left\|U(t-s)\left\{B\left(\partial_{s} n\left(\phi_{0}+\int_{0}^{s} F \mathrm{~d} \tau\right)+n F\right)\right\}\right\|_{L^{4}} \mathrm{~d} s \\
& \leq\left\|U(t) i\left(\Delta \phi_{0}+B\left(n_{0} \phi_{0}\right)\right)\right\|_{L^{4}}+\int_{0}^{t} C|t-s|^{-N / 4}\left\|B\left(\partial_{s} n\left(\phi_{0}+\int_{0}^{s} F \mathrm{~d} \tau\right)+n F\right)\right\|_{L^{4 / 3}} \mathrm{~d} s \\
& \leq\left\|U(t) i\left(\Delta \phi_{0}+B\left(n_{0} \phi_{0}\right)\right)\right\|_{L^{4}}+\int_{0}^{t} C|t-s|^{-N / 4} \\
& \quad \times\left\{\left\|\partial_{s}\right\|_{L^{2}}\left(\left\|\phi_{0}\right\|_{L^{4}}+\int_{0}^{s}\|F\|_{L^{4}} \mathrm{~d} \tau\right)+\|n\|_{L^{2}}\|F\|_{L^{4}}\right\} \mathrm{d} s . \\
& \left\|N_{1}[F, n]\right\|_{L^{8 / N}\left(I ; L^{4}\right)} \\
& \leq \delta a+C\left\{\left\|\frac{\mathrm{d} n \|}{\mathrm{d} t}\right\|_{L^{\infty}\left(I ; L^{2}\right)}\left\|\phi_{0}\right\|_{L^{4}}\left\|\int_{0}^{t}|t-s|^{-N / 4} \mathrm{~d} s\right\|_{L^{8 / N}(I)}\right. \\
& \quad+\left\|\frac{\mathrm{d} n}{\mathrm{~d} t}\right\|_{L^{\infty}\left(I ; L^{2}\right)}\left\|\phi_{0}\right\|_{L^{4}}\left\|\int_{0}^{t}|t-s|^{-N / 4}\left(\int_{0}^{s}\|F\|_{L^{4}} \mathrm{~d} \tau\right) \mathrm{d} s\right\|_{L^{8 / N}(I)} \\
& \left.\quad+\|n\|_{L^{\infty}\left(I ; L^{2}\right)}\left\|\int_{0}^{t}|t-s|^{-N / 4}\right\| F\left\|_{L^{4}} \mathrm{~d} s\right\|_{L^{8 / N}(I)}\right\} .
\end{aligned}
$$


Now

$$
\begin{gathered}
\left\|\int_{0}^{T} T^{-N / 4} \mathrm{~d} s\right\|_{L^{8 / N}(I)}=\left\|T^{1-N / 4}\right\|_{L^{8 / N}(I)}=T^{1-N / 8}, \\
\left\|\int_{0}^{T} T^{-N / 4}\left(\int_{0}^{T}\|F\|_{L^{4}} \mathrm{~d} \tau\right) \mathrm{d} s\right\|_{L^{8 / N}(I)} \leq\left\|T^{2-N / 4}\right\| F\left\|_{L^{4}} \mathrm{~d} s\right\|_{L^{8 / N}(I)}=T^{2-N / 4}\|F\|_{L^{8 / N}\left(I ; L^{4}\right)},
\end{gathered}
$$

and

Hence, we have

$$
\left\|\int_{0}^{T} T^{-N / 4}\right\| F\left\|_{L^{4}} \mathrm{~d} s\right\|_{L^{8 / N}(I)} \leq T^{1-N / 4}\|F\|_{L^{8 / N}\left(I ; L^{4}\right)} .
$$

$$
\begin{aligned}
\left\|N_{1}[F, n]\right\|_{L^{8 / N}\left(I ; L^{4}\right)} & \\
\leq & \delta a+C T^{1-N / 4}\|n\|_{L^{\infty}\left(I ; L^{2}\right)}\|F\|_{L^{8 / N}\left(I ; L^{4}\right)}+C T^{1-N / 8}\left\|\frac{\mathrm{d} n}{\mathrm{~d} t}\right\|_{L^{\infty}\left(I ; L^{2}\right)}\left\|\phi_{0}\right\|_{L^{4}} \\
& +C T^{2-N / 8}\left\|\frac{\mathrm{d} n}{\mathrm{~d} t}\right\|_{L^{\infty}\left(I ; L^{2}\right)}\|F\|_{L^{8 / N}\left(I ; L^{4}\right)} \\
\leq & \delta a+C\left(T^{1-N / 4} \delta a^{2}+T^{1-N / 8} a^{2}+T^{2-N / 4} \delta a^{2}\right) \\
\leq & \delta a+C\left(T^{1-N / 4} a+T^{1-N / 8} \delta^{-1} a+T^{2-N / 4} a\right) \delta a .
\end{aligned}
$$

We choose $T$ sufficiently small so that $C\left(T^{1-N / 4} a+T^{1-N / 8} \delta^{-1} a+T^{2-N / 4} a\right) \leq 1$. Hence

$$
\left\|N_{1}[F, n]\right\|_{L^{8 / N}\left(I ; L^{4}\right)} \leq 2 \delta a .
$$

Using the third lemma and (13), we obtain that

$$
\begin{aligned}
& \left\|N_{1}[F, n]\right\|_{L^{\infty}\left(I ; L^{2}\right)} \\
& \leq\left\|U(t) i\left(\Delta \phi_{0}+B\left(n_{0} \phi_{0}\right)\right)\right\|_{L^{\infty}\left(I ; L^{2}\right)}+C\left\|B\left(\partial_{t} n\left(\phi_{0}+\int_{0}^{t} F \mathrm{~d} s\right)+n F\right)\right\|_{L^{8 /(8-N)}\left(I ; L^{4 / 3}\right)} \\
& \leq a+C\left\|\partial_{t} n\left(\phi_{0}+\int_{0}^{t} F \mathrm{~d} s\right)+n F\right\|_{L^{8 /(8-N)}\left(I ; L^{4 / 3}\right)} \\
& \leq a+C\|\| \partial_{t} n\left\|_{L^{2}}\left(\left\|\phi_{0}\right\|_{L^{4}}+\int_{0}^{t}\|F\|_{L^{4}} \mathrm{~d} s\right)+\right\| n\left\|_{L^{2}}\right\| F\left\|_{L^{4}}\right\|_{L^{8 /(8-N)}(I)} \\
& \leq a+C\left\|\frac{\mathrm{d} n}{\mathrm{~d} t}\right\|_{L^{\infty}\left(I ; L^{2}\right)}\left\|\phi_{0}\right\|_{L^{4}}\|1\|_{L^{8 /(8-N)}(I)}+C\left\|\frac{\mathrm{d} n}{\mathrm{~d} t}\right\|_{L^{\infty}\left(I ; L^{2}\right)}\left\|\int_{0}^{T}\right\| F\left\|_{L^{4}} \mathrm{~d} s\right\|_{L^{8 /(8-N)}(I)} \\
& +C\|n\|_{L^{\infty}\left(I ; L^{2}\right)}\|1\|_{L^{4 /(4-N)}(I)}\|F\|_{L^{8 / N}\left(I ; L^{4}\right)} \text {, } \\
& \leq a+C T^{1-N / 8}\left\|\frac{\mathrm{d} n}{\mathrm{~d} t}\right\|_{L^{\infty}\left(I ; L^{2}\right)}\left\|\phi_{0}\right\|_{L^{4}}+C T^{2-N / 4}\left\|\frac{\mathrm{d} n}{\mathrm{~d} t}\right\|_{L^{\infty}\left(I ; L^{2}\right)}\|F\|_{L^{8 / N}\left(I ; L^{4}\right)} \\
& +C T^{1-N / 4}\|n\|_{L^{\infty}\left(I ; L^{2}\right)}\|F\|_{L^{8 / N}\left(I ; L^{4}\right)} \\
& \leq a+C\left(T^{1-N / 8} a^{2}+T^{2-N / 4} \delta a^{2}+T^{1-N / 4} \delta a^{2}\right), \\
& \leq a+C\left(T^{1-N / 8} a+T^{2-N / 4} \delta a+T^{1-N / 4} \delta a\right) a .
\end{aligned}
$$


We choose $T$ sufficiently small so that $C\left(T^{1-N / 8} a+T^{2-N / 4} \delta a+T^{1-N / 4} \delta a\right) \leq 1$. Hence

$$
\left\|N_{1}[F, n]\right\|_{L^{\infty}\left(I ; L^{2}\right)} \leq 2 a .
$$

In order to treat the second coordinate of $N$, we first estimate $\phi$ in $L^{\infty}\left(I ; H^{2}\right)$,

$$
\begin{gathered}
\phi(t)=(-\Delta+1)^{-1}\left\{i F+B\left(n\left(\phi_{0}+\int_{0}^{t} F \mathrm{~d} s\right)\right)+\left(\phi_{0}+\int_{0}^{t} F \mathrm{~d} s\right)\right\}, \\
(-\Delta+1) \phi(t)=i F+B\left(n\left(\phi_{0}+\int_{0}^{t} F \mathrm{~d} s\right)\right)+\phi_{0}+\int_{0}^{t} F \mathrm{~d} s, \\
\|\phi\|_{H^{2}} \leq\|F\|_{L^{2}}+C\left\|n\left(\phi_{0}+\int_{0}^{t} F \mathrm{~d} s\right)\right\|_{L^{2}}+\left\|\phi_{0}\right\|_{L^{2}}+\int_{0}^{t}\|F\|_{L^{2}} \mathrm{~d} s, \\
\|\phi\|_{L^{\infty}\left(I ; H^{2}\right)} \leq\|F\|_{L^{\infty}\left(I ; L^{2}\right)}+C\|n\|_{L^{\infty}\left(I ; L^{4}\right)}\left(\left\|\phi_{0}\right\|_{L^{4}}+\int_{0}^{T}\|F\|_{L^{4}} \mathrm{~d} s\right)+\left\|\phi_{0}\right\|_{L^{2}}+\int_{0}^{T}\|F\|_{L^{2}} \mathrm{~d} s .
\end{gathered}
$$

Now

$$
\int_{0}^{T}\|F\|_{L^{4}} \mathrm{~d} t \leq C T^{1-N / 8}\|F\|_{L^{8 / N}\left(I ; L^{4}\right)}
$$

then

$$
\begin{gathered}
\|\phi\|_{L^{\infty}\left(I ; H^{2}\right)} \leq C\left(a+a^{2}+T^{1-N / 8} \delta a^{2}+T a\right) . \\
N_{2}[F, n](t)=\cos (\omega t) n_{0}+\omega^{-1} \sin (\omega t) n_{1}+\int_{0}^{t} \omega^{-1} \sin (\omega(t-s)) \Delta|\phi(s)|^{2} \mathrm{~d} s .
\end{gathered}
$$

Using lemma 4 and (23), we have

$$
\begin{aligned}
\left\|N_{2}[F, n](t)\right\|_{H^{\prime}}= & \left\|(1-\Delta)^{1 / 2} N_{2}[F, n](t)\right\|_{L^{2}} \\
\leq & \left\|\left(1+\omega^{2}\right)^{1 / 2} \cos (\omega t) n_{0}\right\|_{L^{2}}+\left\|\left(1+\omega^{2}\right)^{1 / 2} \omega^{-1} \sin (\omega t) n_{1}\right\|_{L^{2}} \\
& +\int_{0}^{t}\left\|\left(1+\omega^{2}\right)^{1 / 2} \omega^{-1} \sin (\omega(t-s)) \Delta|\phi(s)|^{2}\right\|_{L^{2}} \mathrm{~d} s \\
\leq & \left\|n_{0}\right\|_{H^{1}}+(1+t)\left\|n_{1}\right\|_{L^{2}}+\int_{0}^{t}(1+(t-s))\left\|\Delta|\phi(s)|^{2}\right\|_{L^{2}} \mathrm{~d} s \\
\leq & (1+T) a+\int_{0}^{T}(1+T)\left\|\Delta|\phi(s)|^{2}\right\|_{L^{2}} \mathrm{~d} s \\
\leq & (1+T) a+T(1+T)\left\|\Delta|\phi|^{2}\right\|_{L^{\infty}\left(I ; L^{2}\right)} \\
\leq & (1+T) a+C T(1+T)\|\phi\|_{L^{\infty}\left(I ; H^{2}\right)}^{2}
\end{aligned}
$$

because $\left\|\Delta|\phi|^{2}\right\|_{L^{\infty}\left(I ; L^{2}\right)} \leq C\|\phi\|_{L^{\infty}\left(I ; H^{2}\right)}^{2}$,

$$
\left\|N_{2}[F, n]\right\|_{L^{\infty}\left(I ; H^{1}\right)} \leq a+\left[T+C T(1+T)\left(1+a+T^{1-N / 8} \delta a+T\right)^{2} a\right] a .
$$


We choose $T$ sufficiently small so that $T+C T(1+T)\left(1+a+T^{1-N / 8} \delta a+T\right)^{2} a \leq 1$. Hence

$$
\begin{gathered}
\left\|N_{2}[F, n]\right\|_{L^{\infty}\left(I ; H^{1}\right)} \leq 2 a . \\
\frac{\mathrm{d}}{\mathrm{d} t} N_{2}[F, n](t)=-\omega \sin (\omega t) n_{0}+\cos (\omega t) n_{1}+\int_{0}^{t} \cos (\omega(t-s)) \Delta|\phi(s)|^{2} \mathrm{~d} s .
\end{gathered}
$$

Using once more lemma 4 and (23),

$$
\begin{aligned}
\left\|\frac{\mathrm{d}}{\mathrm{d} t} N_{2}[F, n](t)\right\|_{L^{\infty}\left(I ; L^{2}\right)} & \leq\left\|n_{0}\right\|_{H^{1}}+\left\|n_{1}\right\|_{L^{2}}+\int_{0}^{T}\left\|\Delta|\phi(s)|^{2}\right\|_{L^{2}} \mathrm{~d} s \\
& \leq\left\|n_{0}\right\|_{H^{1}}+\left\|n_{1}\right\|_{L^{2}}+C T\|\phi\|_{L^{\infty}\left(I ; H^{2}\right)}^{2} \\
& \leq a+C T\left(1+a+T^{1-N / 8} \delta a+T\right)^{2} a . a .
\end{aligned}
$$

We choose $T$ sufficiently small so that $C T\left(1+a+T^{1-N / 8} \delta a+T\right)^{2} a \leq 1$. Hence

$$
\left\|\frac{\mathrm{d}}{\mathrm{d} t} N_{2}[F, n]\right\|_{L^{\infty}\left(I ; L^{2}\right)} \leq 2 a .
$$

Collecting the four results (21), (22), (24), (25), we concude that $N$ maps $Y$ into itself (for $T$ small).

Second step. $N$ is a contraction in $Y$, i.e. $\forall(F, n),\left(F^{\prime}, n^{\prime}\right) \in Y$ and $T$ sufficiently small

$$
\left\|N[F, n]-N\left[F^{\prime}, n^{\prime}\right]\right\| \leq \frac{1}{2}\|\|(F, n)-\left(F^{\prime}, n^{\prime}\right) \|
$$

where $\|\cdot\|$ denotes the natural the norm of $X$.

The computation which follow are essentially the same as in the first step.

$$
\begin{aligned}
& N_{1}[F, n](t)-N_{1}\left[F^{\prime}, n^{\prime}\right](t) \\
& =i \int_{0}^{t} U(t-s)\left\{B\left(\partial_{s} n\left(\phi_{0}+\int_{0}^{s} F \mathrm{~d} \tau\right)+n F\right)-B\left(\partial_{s} n^{\prime}\left(\phi_{0}+\int_{0}^{s} F^{\prime} \mathrm{d} \tau\right)+n^{\prime} F^{\prime}\right)\right\} \mathrm{d} s, \\
& N_{1}[F, n]-N_{1}\left[F^{\prime}, n^{\prime}\right] \\
& =i \int_{0}^{t} U(t-s)\left\{B\left(\left(\partial_{s} n-\partial_{s} n^{\prime}\right) \phi_{0}\right)+B\left(\left(\partial_{s} n-\partial_{s} n^{\prime}\right) \int_{0}^{s} F \mathrm{~d} \tau+\partial_{s} n^{\prime}\left(\int_{0}^{s}\left(F-F^{\prime}\right) \mathrm{d} \tau\right)\right)\right. \\
& \left.\quad+B\left(\left(n-n^{\prime}\right) F+n^{\prime}\left(F-F^{\prime}\right)\right)\right\} \mathrm{d} s, \\
& \left\|N_{1}[F, n](t)-N_{1}\left[F^{\prime}, n^{\prime}\right](t)\right\|_{L^{4}} \\
& \quad \int_{0}^{t}(4 \pi|t-s|)^{-N / 4}\left\{\left\|\partial_{s} n-\partial_{s} n^{\prime}\right\|_{L^{2}}\left\|\phi_{0}\right\|_{L^{4}}+\left\|\partial_{s} n-\partial_{s} n^{\prime}\right\|_{L^{2}} \int_{0}^{T}\|F\|_{L^{4}} \mathrm{~d} \tau\right. \\
& \left.\quad+\left\|\partial_{s} n^{\prime}\right\|_{L^{2}} \int_{0}^{T}\left\|F-F^{\prime}\right\|_{L^{4}} \mathrm{~d} \tau+\left\|n-n^{\prime}\right\|_{L^{2}}\|F\|_{L^{4}}+\left\|n^{\prime}\right\|_{L^{2}}\left\|F-F^{\prime}\right\|_{L^{4}}\right\} \mathrm{d} s,
\end{aligned}
$$




$$
\begin{aligned}
\| N_{1}[F, n] & -N_{1}\left[F^{\prime}, n^{\prime}\right] \|_{L^{8 / N}\left(I ; L^{4}\right)} \\
\leq & C\left\{\left\|\int_{0}^{t} T^{-N / 4}\right\| \partial_{s} n-\partial_{s} n^{\prime}\left\|_{L^{2}}\right\| \phi_{0}\left\|_{L^{4}} \mathrm{~d} s\right\|_{L^{8 / N}(I)}\right. \\
& +\left\|\int_{0}^{t} T^{-N / 4}\right\| \partial_{s} n^{\prime}\left\|_{L^{2}} \int_{0}^{T}\right\| F-F^{\prime}\left\|_{L^{4}} \mathrm{~d} \tau\right\|_{L^{8 / N}(I)} \\
& +\left\|\int_{0}^{t} T^{-N / 4}\right\| \partial_{s} n-\partial_{s} n^{\prime}\left\|_{L^{2}} \int_{0}^{T}\right\| F\left\|_{L^{4}} \mathrm{~d} \tau\right\|_{L^{8 / N}(I)} \\
& +\left\|\int_{0}^{t} T^{-N / 4}\right\| n-n^{\prime}\left\|_{L^{2}} \int_{0}^{T}\right\| F\left\|_{L^{4}} \mathrm{~d} \tau\right\|_{L^{8 / N}(I)} \\
& \left.+\left\|\int_{0}^{t} T^{-N / 4}\right\| n^{\prime}\left\|_{L^{2}} \int_{0}^{T}\right\| F-F^{\prime}\left\|_{L^{4}} \mathrm{~d} \tau\right\|_{L^{8 / N}(I)}\right\} \\
\leq & C\left\{T^{1-N / 8}\left\|\frac{\mathrm{d}}{\mathrm{d} t}\left(n-n^{\prime}\right)\right\|_{L^{\infty}\left(I ; L^{2}\right)}\left\|\phi_{0}\right\|_{L^{4}}+T^{2-N / 4}\left\|\frac{\mathrm{d}}{\mathrm{d} t}\left(n-n^{\prime}\right)\right\|_{L^{\infty}\left(I ; L^{2}\right)}\|F\|_{L^{8 / N}\left(I ; L^{4}\right)}\right. \\
& \left.+T^{1-N / 4}\left\|n^{\prime}\right\|_{L^{\infty}\left(I ; L^{2}\right)}\left\|F-F^{\prime}\right\|_{L^{8 / N}\left(I ; L^{4}\right)}\right\}_{L^{\infty}\left(I ; L^{2}\right)}\left\|F-F^{\prime}\right\|_{L^{8 / N}\left(I ; L^{4}\right)}+T^{1-N / 4}\left\|n-n^{\prime}\right\|_{L^{\infty}\left(I ; L^{2}\right)}\|F\|_{L^{8 / N}\left(I ; L^{4}\right)} \\
& +T^{2-N / 4}\left\|\frac{\mathrm{d}}{\mathrm{d} t} n^{\prime}\right\| \\
& \\
& \\
&
\end{aligned}
$$

We choose $T$ sufficiently small so that $C\left\{T^{1-N / 8} a+T^{2-N / 4}(\delta a+a)+T^{1-N / 4}(\delta a+a)\right\} \leq \frac{1}{8}$. Hence

$$
\left\|N_{1}[F, n]-N_{1}\left[F^{\prime}, n^{\prime}\right]\right\|_{L^{8 / N}\left(I ; L^{4}\right)} \leq \frac{1}{8}\left\|(F, n)-\left(F^{\prime}, n^{\prime}\right)\right\| .
$$

$$
\begin{aligned}
\left\|N_{1}[F, n]-N_{1}\left[F^{\prime}, n^{\prime}\right]\right\|_{L^{\infty}\left(I ; L^{2}\right)} & \\
\leq & C \| B\left(\left(\partial_{t} n-\partial_{t} n^{\prime}\right) \phi_{0}\right)+B\left(\left(\partial_{t} n-\partial_{t} n^{\prime}\right) \int_{0}^{t} F \mathrm{~d} s+\left(\partial_{t} n^{\prime}\left(\int_{0}^{t}\left(F-F^{\prime}\right) \mathrm{d} s\right)\right)\right) \\
& +B\left(\left(n-n^{\prime}\right) F+n^{\prime}\left(F-F^{\prime}\right)\right) \|_{L^{8 /(8-N)}\left(I ; L^{4 / 3}\right)} \\
\leq & C\left\{\left\|\left(\partial_{t} n-\partial_{t} n^{\prime}\right) \phi_{0}\right\|_{L^{8 /(8-N)}\left(I ; L^{4 / 3}\right)}+\left\|\left(\partial_{t} n-\partial_{t} n^{\prime}\right) \int_{0}^{t} F \mathrm{~d} s\right\|_{L^{8 /(8-N)}\left(I ; L^{4 / 3}\right)}+\left\|\left(n-n^{\prime}\right) F\right\|_{L^{8 /(8-N)}\left(I ; L^{4 / 3}\right)}\right. \\
& +\left\|\partial_{t} n^{\prime} \int_{0}^{t}\left(F-F^{\prime}\right) \mathrm{d} s\right\|_{L^{8 /(8-N)}\left(I ; L^{4 / 3}\right)} \\
& \left.+\left\|n^{\prime}\left(F-F^{\prime}\right)\right\|_{L^{8 /(8-N)}\left(I ; L^{4 / 3}\right)}\right\}
\end{aligned}
$$




$$
\begin{aligned}
\leq & C\left\{\|\| \partial_{t} n-\partial_{t} n^{\prime}\left\|_{L^{2}}\right\| \phi_{0}\left\|_{L^{4}}\right\|_{L^{8 /(8-N)}(I)}+\|\| \partial_{t} n-\partial_{t} n^{\prime}\left\|_{L^{2}} \int_{0}^{T}\right\| F\left\|_{L^{4}} \mathrm{~d} s\right\|_{L^{8 /(8-N)}(I)}\right. \\
& +\|\| \partial_{\mathrm{I}} n^{\prime}\left\|_{L^{2}} \int_{0}^{T}\right\| F-F^{\prime}\left\|_{L^{4}} \mathrm{~d} s\right\|_{L^{8 / 8-N)}(I)}+\|\| n-n^{\prime}\left\|_{L^{2}}\right\| F\left\|_{L^{4}}\right\|_{L^{8 /(8-N)}(I)} \\
& \left.+\|\| n^{\prime}\left\|_{L^{2}}\right\| F-F^{\prime}\left\|_{L^{4}}\right\|_{L^{8 /(8-N)}(I)}\right\} \\
\leq & C\left\{T^{1-N / 8}\left\|\frac{\mathrm{d}}{\mathrm{d} t}\left(n-n^{\prime}\right)\right\|_{L^{\infty}\left(I ; L^{2}\right)}\left\|\phi_{0}\right\|_{L^{4}}+T^{2-N / 4}\left\|\frac{\mathrm{d}}{\mathrm{d} t}\left(n-n^{\prime}\right)\right\|_{L^{\infty}\left(I ; L^{2}\right)}\|F\|_{L^{8 / N}\left(I ; L^{4}\right)}\right. \\
& +T^{2-N / 4}\left\|\frac{\mathrm{d}}{\mathrm{d} t} n^{\prime}\right\|_{L^{\infty}\left(I ; L^{2}\right)}\left\|F-F^{\prime}\right\|_{L^{8 / N}\left(I ; L^{4}\right)}+T^{1-N / 4}\left\|n-n^{\prime}\right\|_{L^{\infty}\left(I ; L^{2}\right)}\|F\|_{L^{8 / N}\left(I ; L^{4}\right)} \\
& \left.+T^{1-N / 4}\left\|n^{\prime}\right\|_{L^{\infty}\left(I ; L^{2}\right)}\left\|F-F^{\prime}\right\|_{L^{8 / N}\left(I ; L^{4}\right)}\right\} \\
\leq & C\left\{T^{1-N / 8} a+T^{2-N / 4}(\delta a+a)+T^{1-N / 4}(\delta a+a)\right\}\left\|(F, n)-\left(F^{\prime}, n^{\prime}\right)\right\| .
\end{aligned}
$$

We choose $T$ sufficiently small so that $C\left(T^{1-N / 8} a+T^{2-N / 4} \delta a+T^{1-N / 4} \delta a\right) \leq \frac{1}{8}$. Hence

$$
\left\|N_{1}[F, n]-N_{1}\left[F^{\prime}, n^{\prime}\right]\right\|_{L^{\infty}\left(I ; L^{2}\right)} \leq \frac{1}{8}\left\|(F, n)-\left(F^{\prime}, n^{\prime}\right)\right\| .
$$

$$
\begin{aligned}
\phi(t)- & \phi^{\prime}(t) \\
= & (-\Delta+1)^{-1}\left\{i\left(F-F^{\prime}\right)+B\left(\left(n-n^{\prime}\right) \phi_{0}\right)+B\left(\left(n-n^{\prime}\right) \int_{0}^{t} F \mathrm{~d} s\right)\right. \\
& \left.+B\left(n^{\prime} \int_{0}^{t}\left(F-F^{\prime}\right) \mathrm{d} s\right)+\int_{0}^{t}\left(F-F^{\prime}\right) \mathrm{d} s\right\},
\end{aligned}
$$

$\left\|\phi(t)-\phi^{\prime}(t)\right\|_{H^{2}}$

$$
\begin{aligned}
\leq & C\left\{\left\|F-F^{\prime}\right\|_{L^{2}}+\left\|\left(n-n^{\prime}\right) \phi_{0}\right\|_{L^{2}}+\left\|\left(n-n^{\prime}\right) \int_{0}^{T} F \mathrm{~d} s\right\|_{L^{2}}+\left\|n^{\prime} \int_{0}^{T}\left(F-F^{\prime}\right) \mathrm{d} s\right\|_{L^{2}}\right. \\
& \left.+\left\|\int_{0}^{T}\left(F-F^{\prime}\right) \mathrm{d} s\right\|_{L^{2}}\right\}, \\
\| \phi- & \phi^{\prime} \|_{L^{\infty}\left(I ; H^{2}\right)} \\
\leq & C\left\{\left\|F-F^{\prime}\right\|_{L^{\infty}\left(I ; L^{2}\right)}+\left\|n-n^{\prime}\right\|_{L^{\infty}\left(I ; L^{4}\right)}\left\|\phi_{0}\right\|_{L^{4}}+\left\|n-n^{\prime}\right\|_{L^{\infty}\left(I ; L^{4}\right)} \int_{0}^{T}\|F\|_{L^{4}} \mathrm{~d} s\right. \\
& \left.+\left\|n^{\prime}\right\|_{L^{\infty}\left(I ; L^{4}\right)} \int_{0}^{T}\left\|F-F^{\prime}\right\|_{L^{4}} \mathrm{~d} s+\int_{0}^{T}\left\|F-F^{\prime}\right\|_{L^{2}} \mathrm{~d} s\right\} .
\end{aligned}
$$


So

$$
\begin{gathered}
\left\|\phi-\phi^{\prime}\right\|_{L^{\infty}\left(I ; H^{2}\right)} \leq C\left\{1+a+T^{1-N / 8}(\delta a+a)+T\right\}\left\|(F, n)-\left(F^{\prime}, n^{\prime}\right)\right\| \\
N_{2}[F, n](t)-N_{2}\left[F^{\prime}, n^{\prime}\right](t)=\int_{0}^{t} \omega^{-1} \sin (\omega(t-s))\left(\Delta|\phi(s)|^{2}-\Delta\left|\phi^{\prime}(s)\right|^{2} \mathrm{~d} s\right. \\
\left\|N_{2}[F, n](t)-N_{2}\left[F^{\prime}, n^{\prime}\right](t)\right\|_{H^{\prime}} \\
\leq \int_{0}^{t}\left\|\left(1+\omega^{2}\right)^{1 / 2} \omega^{-1} \sin (\omega(t-s))\left(\Delta|\phi|^{2}-\Delta\left|\phi^{\prime}\right|^{2}\right)\right\|_{L^{2}} \mathrm{~d} s \\
\leq \int_{0}^{t}(1+(t-s))\left\|\Delta\left(|\phi(s)|^{2}-\left|\phi^{\prime}(s)\right|^{2}\right)\right\|_{L^{2}} \mathrm{~d} s
\end{gathered}
$$

We have $\left\|\Delta\left(|\phi|^{2}-\left|\phi^{\prime}\right|^{2}\right)\right\|_{L^{2}} \leq C\left(\|\phi\|_{H^{2}}+\left\|\phi^{\prime}\right\|_{H^{2}}\right)\left\|\phi-\phi^{\prime}\right\|_{H^{2}}$, therefore,

$$
\begin{gathered}
\left\|N_{2}[F, n](t)-N_{2}\left[F^{\prime}, n^{\prime}\right](t)\right\|_{H^{1}} \leq C T(1+T)\left(\|\phi(t)\|_{H^{2}}+\left\|\phi^{\prime}(t)\right\|_{\left.H^{2}\right)}\left\|\left(\phi-\phi^{\prime}\right)(t)\right\|_{H^{2}}\right. \\
\left\|N_{2}[F, n]-N_{2}\left[F^{\prime}, n^{\prime}\right]\right\|_{L^{\infty}\left(I ; H^{1}\right)} \\
\leq C T(1+T)\left(\|\phi\|_{L^{\infty}\left(I ; H^{2}\right)}+\left\|\phi^{\prime}\right\|_{L^{\infty}\left(I ; H^{2}\right)}\right)\left\|\phi-\phi^{\prime}\right\|_{L^{\infty}\left(I ; H^{2}\right)} \\
\leq C T(1+T)\left(a+a^{2}+T^{1-N / 8} \delta a^{2}+T a\right) \\
\times\left(1+a+T^{1-N / 8}(\delta a+a)+T\right)\left\|(F, n)-\left(F^{\prime}, n^{\prime}\right)\right\| .
\end{gathered}
$$

We choose $T$ sufficiently small so that

Hence

$$
C T(1+T)\left(a+a^{2}+T^{1-N / 8} \delta a^{2}+T a\right)\left(1+a+T^{1-N / 8}(\delta a+a)+T\right) \leq \frac{1}{8} .
$$

$$
\begin{gathered}
\left\|N_{2}[F, n]-N_{2}\left[F^{\prime}, n^{\prime}\right]\right\|_{L^{\infty}\left(I ; H^{1}\right)} \leq \frac{1}{8}\left\|(F, n)-\left(F^{\prime}, n^{\prime}\right)\right\| \\
\frac{\mathrm{d}}{\mathrm{d} t} N_{2}[F, n](t)-\frac{\mathrm{d}}{\mathrm{d} t} N_{2}\left[F^{\prime}, n^{\prime}\right](t)=\int_{0}^{t} \cos (\omega(t-s)) \Delta\left(|\phi(s)|^{2}-\left(\left|\phi^{\prime}(s)\right|^{2}\right) \mathrm{d} s\right. \\
\left\|\frac{\mathrm{d}}{\mathrm{d} t} N_{2}[F, n](t)-\frac{\mathrm{d}}{\mathrm{d} t} N_{2}\left[F^{\prime}, n^{\prime}\right](t)\right\|_{L^{2}} \leq \int_{0}^{t} \| \Delta\left(|\phi(s)|^{2}-\left(\left|\phi^{\prime}(s)\right|^{2}\right) \|_{L^{2}} \mathrm{~d} s\right. \\
\left\|\frac{\mathrm{d}}{\mathrm{d} t}\left(N_{2}[F, n]-N_{2}\left[F^{\prime}, n^{\prime}\right]\right)\right\|_{L^{\infty}\left(I ; L^{2}\right)} \\
\leq C T\left(\|\phi\|_{L^{\infty}\left(I ; H^{2}\right)}+\left\|\phi^{\prime}\right\|_{L^{\infty}\left(I ; H^{2}\right)}\right)\left\|\phi-\phi^{\prime}\right\|_{L^{\infty}\left(I ; H^{2}\right)} \\
\leq C T\left(a+a^{2}+T^{1-N / 8} \delta a^{2}+T a\right)\left(1+a+T^{1-N / 8}(\delta a+a)+T\right)\left\|(F, n)-\left(F^{\prime}, n^{\prime}\right)\right\|
\end{gathered}
$$

We choose $T$ sufficiently small so that

$$
C T\left(a+a^{2}+T^{1-N / 8} \delta a^{2}+T a\right)\left(1+a+T^{1-N / 8}(\delta a+a)+T\right) \leq \frac{1}{8} .
$$


Hence

$$
\left\|\frac{\mathrm{d}}{\mathrm{d} t}\left(N_{2}[F, n]-N_{2}\left[F^{\prime}, n^{\prime}\right]\right)\right\|_{L^{\infty}\left(I ; L^{2}\right)} \leq \frac{1}{8}\left\|(F, n)-\left(F^{\prime}, n^{\prime}\right)\right\| .
$$

Summing up all the above estimates (26), (27), (29), (30), we obtain

$$
\left\|N(F, n)-N\left(F^{\prime}, n^{\prime}\right)\right\| \leq \frac{1}{2}\left\|(F, n)-\left(F^{\prime}, n^{\prime}\right)\right\| .
$$

$N$ is also a contraction from $Y$ into $Y$ for $T$ sufficiently small. $N$ has also a unique fixed point in $Y$ such that

$$
\left\{\begin{array}{l}
F(t)=U(t) F_{0}+i \int_{0}^{t} U(t-s)\left\{B\left(\partial_{s} n\left(\phi_{0}+\int_{0}^{s} F \mathrm{~d} \tau\right)+n F\right)\right\} \mathrm{d} s \\
n(t)=\cos (\omega t) n_{0}+\omega^{-1} \sin (\omega t) n_{1}+\int_{0}^{t} \omega^{-1} \sin (\omega(t-s)) \Delta|\phi(s)|^{2} \mathrm{~d} s \\
\phi(t)=(-\Delta+1)^{-1}\left\{i F+B\left(n\left(\phi_{0}+\int_{0}^{t} F \mathrm{~d} s\right)\right)+\left(\phi_{0}+\int_{0}^{t} F \mathrm{~d} s\right)\right\} .
\end{array}\right.
$$

We obtain immediately that

$$
\left\{\begin{array}{l}
F(0)=i\left(\Delta \phi_{0}+B\left(n_{0} \phi_{0}\right)\right)=F_{0}, \\
n(0)=n_{0}, \quad \partial_{t}(0)=n_{1}, \\
\phi(0)=\phi_{0} .
\end{array}\right.
$$

$(F, n, \phi)$ satisfies (16), (17), in the integral form. $F_{0} \in L^{2}$ so $U(t) F_{0} \in \mathcal{C}\left(I ; L^{2}\right) \cap \mathcal{C}^{1}\left(I ; H^{-2}\right)$, and $\partial_{t} U(t) F_{0}=i U(t) \Delta F_{0}=i \Delta\left(U(t) F_{0}\right)$. As $L^{4 / 3} \hookrightarrow H^{-1}$,

$$
B\left(\partial_{s} n\left(\phi_{0}+\int_{0}^{s} F \mathrm{~d} \tau\right)+n F\right) \in L^{1}\left(I ; H^{-1}\right)
$$

hence

$$
\int_{0}^{t} U(t-s)\left\{B\left(\partial_{s} n\left(\phi_{0}+\int_{0}^{s} F \mathrm{~d} \tau\right)+n F\right)\right\} \mathrm{d} s \in \mathcal{C}\left(I ; H^{-1}\right) \cap W^{1,1}\left(I ; H^{-3}\right)
$$

and

$$
\begin{aligned}
& \partial_{t} \int_{0}^{t} U(t-s)\left\{B\left(\partial_{s} n\left(\phi_{0}+\int_{0}^{s} F \mathrm{~d} \tau\right)+n F\right)\right\} \mathrm{d} s \\
& =i \Delta \int_{0}^{t} U(t-s)\left\{B\left(\partial_{s} n\left(\phi_{0}+\int_{0}^{s} F \mathrm{~d} \tau\right)+n F\right)\right\} \mathrm{d} s+B\left(\partial_{t} n\left(\phi_{0}+\int_{0}^{t} F \mathrm{~d} \tau\right)+n F\right) \\
& =i \int_{0}^{t} U(t-s) \Delta\left\{B\left(\partial_{s} n\left(\phi_{0}+\int_{0}^{s} F \mathrm{~d} \tau\right)+n F\right)\right\} \mathrm{d} s+B\left(\partial_{t} n\left(\phi_{0}+\int_{0}^{t} F \mathrm{~d} \tau\right)+n F\right),
\end{aligned}
$$


so that

$$
\begin{aligned}
\partial_{t} F(t)= & i \Delta\left(U(t) F_{0}+i \int_{0}^{t} U(t-s)\left\{B\left(\partial_{s} n\left(\phi_{0}+\int_{0}^{s} F \mathrm{~d} \tau\right)+n F\right)\right\} \mathrm{d} s\right) \\
& +i B\left(\partial_{t} n\left(\phi_{0}+\int_{0}^{t} F \mathrm{~d} \tau\right)+n F\right) .
\end{aligned}
$$

That is

$$
\partial_{t} F(t)=i \Delta F(t)+i B\left(\partial_{t} n\left(\phi_{0}+\int_{0}^{t} F \mathrm{~d} \tau\right)+n F\right)
$$

i.e.

$$
i \dot{F}+\Delta F=-B\left(\partial_{t} n\left(\phi_{0}+\int_{0}^{t} F \mathrm{~d} \tau\right)+N F\right) .
$$

Furthermore, $n_{0} \in H^{1}, n_{1} \in L^{2}$, and $\Delta|\phi|^{2} \in L^{2}\left(0, T ; L^{2}\right)$,

$$
n(t) \in \mathcal{C}\left([0, T] ; H^{1}\right), \quad \partial_{t} n(t) \in \mathcal{C}\left([0, T] ; L^{2}\right) \quad \text { and } \quad \partial_{t^{2}} n(t) \in \mathcal{C}\left([0, T] ; H^{-1}\right)
$$

and in $H^{-1}$ we have

$$
\partial_{t} 2 n-\Delta n=\Delta|\phi|^{2}
$$

$(F, n, \phi)$ also verifies the system (16), (17) with the equalities in $H^{-1}$.

We shall now show that

$$
\left\{\begin{array}{l}
F(t) \in\left[\bigcap_{j=0}^{1} \mathcal{e}^{j}\left([0, T] ; H^{-2 j}\right)\right] \cap L^{8 / N}\left(0, T ; L^{4}\right), \\
n(t) \in \bigcap_{j=0}^{2} \mathcal{C}^{j}\left([0, T] ; H^{1-j}\right), \\
\phi(t) \in \mathcal{C}\left([0, T] ; H^{2}\right) .
\end{array}\right.
$$

We know, in fact, that if $1 \leq q^{\prime}, r^{\prime} \leq 2,2 \leq q<\alpha(N)$ and $(N / 2-N / q) r=2$, then

$$
\int_{0}^{t} U(t-s) f(s) \mathrm{d} s \in \mathbb{C}^{1}\left(0, T ; L^{2}\right) \quad \text { if } f \in L^{r^{\prime}}\left(I ; L^{q^{\prime}}\right) .
$$

Here we have $F_{0} \in L^{2}$ and

$$
B\left(\partial_{t} n\left(\phi_{0}+\int_{0}^{t} F \mathrm{~d} \tau\right)+n F\right) \in L^{8 /(8-N)}\left(I ; L^{4 / 3}\right),
$$

so that $U(t) F_{0} \in \mathcal{C}\left(0, T ; L^{2}\right) \cap \mathcal{C}^{1}\left(0, T ; H^{-2}\right)$ (cf. Kato [14]),

$$
\int_{0}^{t} U(t-s)\left\{B\left(\partial_{s} n\left(\phi_{0}+\int_{0}^{s} F \mathrm{~d} \tau\right)+n F\right)\right\} \mathrm{d} s \in \mathcal{C}\left(0, T ; L^{2}\right)
$$

so that $F(t) \in \mathcal{C}\left(0, T ; L^{2}\right)$. 
We have already seen that $n(t) \in \bigcap_{j=0}^{2} \mathrm{e}^{j}\left(0, T ; H^{1-j}\right)$. On the other hand,

$$
B\left(\partial_{t} n\left(\phi_{0}+\int_{0}^{t} F \mathrm{~d} \tau\right)+n F\right) \in \mathcal{e}\left(0, T ; L^{4 / 3}\right) \subset \mathcal{e}\left(0, T ; H^{-1}\right)
$$

and $\Delta F(t) \in \mathcal{C}\left(0, T ; H^{-2}\right)$, so that $\partial_{t} F(t) \in \mathcal{C}\left(0, T ; H^{-2}\right)$, and

$$
(-\Delta+1) \phi(t)=i F+B\left(n\left(\phi_{0}+\int_{0}^{t} F \mathrm{~d} s\right)\right)+\left(\phi_{0}+\int_{0}^{t} F \mathrm{~d} s\right) \in \mathcal{C}\left(0, T ; L^{2}\right),
$$

so that $\phi(t) \in \mathcal{C}\left([0, T] ; H^{2}\right)$. Then we have the system (33).

This enables us to differentiate once more in time the equation

$$
(-\Delta+1) \phi(t)=i F+B\left(n\left(\phi_{0}+\int_{0}^{t} F \mathrm{~d} s\right)\right)+\left(\phi_{0}+\int_{0}^{t} F \mathrm{~d} s\right) .
$$

We obtain

$$
(-\Delta+1) \frac{\partial \phi}{\partial t}(t)=i \frac{\partial F}{\partial t}(t)+B\left(\frac{\partial n}{\partial t}\left(\phi_{0}+\int_{0}^{t} F \mathrm{~d} s\right)+n F(t)\right)+F(t)
$$

in $H^{-2}$.

On the other hand,

$$
\partial_{t} F(t)=i \Delta F(t)+i B\left(\partial_{t} n\left(\phi_{0}+\int_{0}^{t} F \mathrm{~d} s\right)+n F\right)
$$

thus

$$
(-\Delta+1) F=i \dot{F}+B\left(\partial_{t} n\left(\phi_{0}+\int_{0}^{t} F \mathrm{~d} s\right)+n F\right)+F .
$$

This yields $(\partial / \partial t) \phi(t)=F(t)$ in $H^{-2}$.

Furthermore,

$$
\frac{\partial}{\partial t} \phi(t)=(-\Delta+1)^{-1}\left(i \frac{\partial F}{\partial t}+B\left(\frac{\partial n}{\partial t}\left(\phi_{0}+\int_{0}^{t} F \mathrm{~d} s\right)+n F(t)\right)+F(t)\right) .
$$

The right-hand term belongs to $\mathcal{C}\left(0, T ; L^{2}\right)$ so that $\phi(t) \in \mathcal{C}^{1}\left(0, T ; L^{2}\right)$,

$$
(-\Delta+1) \phi(t)=i F+B\left(n\left(\phi_{0}+\int_{0}^{t} F \mathrm{~d} s\right)\right)+\left(\phi_{0}+\int_{0}^{t} F \mathrm{~d} s\right) .
$$

As we know that $\int_{0}^{t} F \mathrm{~d} s=\phi(t)-\phi_{0}$, therefore,

$$
(-\Delta+1) \phi(t)=i \frac{\partial \phi}{\partial t}+B(n \phi)+\phi
$$

which yields

$$
i \frac{\partial \phi}{\partial t}+\Delta \phi=-B(n \phi) .
$$


This gives us the existence of a solution of (15). This solution verifies

$$
\begin{aligned}
\left\|\frac{\partial \phi}{\partial t}\right\|_{L^{\infty}\left(I ; L^{2}\right)} & \leq 2 a, \\
\left\|\frac{\partial \phi}{\partial t}\right\|_{L^{8 / N}\left(I ; L^{4}\right)} & \leq 2 \delta a, \\
\|n\|_{L^{\infty}\left(I ; H^{1}\right)} & \leq 2 a, \\
\left\|\frac{\partial n}{\partial t}\right\|_{L^{\infty}\left(I ; L^{2}\right)} & \leq 2 a .
\end{aligned}
$$

On the other hand, $i \dot{\phi}+\Delta \phi=-B(n \phi)$, this gives us the conservation law

$$
\|\phi(t)\|_{L^{2}}=\left\|\phi_{0}\right\|_{L^{2}} \leq a .
$$

A second conservation law may be obtained by setting

$$
-\Delta \Phi=\frac{\partial n}{\partial t}
$$

Then we have

$$
\int\left(|\nabla \phi(t)|^{2}+n(t)|\phi(t)|^{2}+\frac{1}{2}(\nabla \Phi)^{2}+\frac{1}{2} n^{2}(t)\right)=\text { const. }
$$

There only remains to prove the uniqueness of $(\phi, n)$ in a convenient space, which is equivalent to show the uniqueness of $(F, n)$ in $X$. For the time being, we only know that $(F, n)$ is unique in $Y$.

If we have two solutions to the problem, $(F, n)$ and $\left(F^{\prime}, n^{\prime}\right)$, we may associate a maximal time of existence in $X\left(T\right.$ and $\left.T^{\prime}\right)$, and a value $a$ and $a^{\prime}$ such that

$$
\begin{array}{rlrl}
\|F\|_{L^{\infty}\left(I ; L^{2}\right)} & \leq 2 a, & \left\|F^{\prime}\right\|_{L^{\infty}\left(0, T^{\prime} ; L^{2}\right)} & \leq 2 a^{\prime}, \\
\|F\|_{L^{8 / N}\left(I ; L^{4}\right)} & \leq 2 \delta a, & \left\|F^{\prime}\right\|_{L^{8 / N}\left(0, T^{\prime} ; L^{4}\right)} \leq 2 \delta a^{\prime}, \\
\|n\|_{L^{\infty}\left(0, T ; H^{1}\right)} & \leq 2 a, & \left\|n^{\prime}\right\|_{L^{\infty}\left(0, T^{\prime} ; H^{1}\right)} \leq 2 a^{\prime}, \\
\left\|n_{t}\right\|_{L^{\infty}\left(I ; L^{2}\right)} \leq 2 a, & \left\|n_{t}^{\prime}\right\|_{L^{\infty}\left(0, T^{\prime} ; L^{2}\right)} \leq 2 a^{\prime},
\end{array}
$$

with

$$
\max \left\{\left\|\phi_{0}\right\|_{L^{2}},\left\|\phi_{0}\right\|_{L^{4}},\left\|\Delta \phi_{0}+B\left(n_{0} \phi_{0}\right)\right\|_{L^{2}},\left\|n_{0}\right\|_{H^{1}}+\left\|n_{1}\right\|_{L^{2}}\right\} \leq a \text { and } a^{\prime} \text {. }
$$

We set $\tau=\min \left(T, T^{\prime}\right)$ and $\alpha=\max \left(a, a^{\prime}\right)$. Then we have the above estimations with $\tau$ and $\alpha$. Following exactly the same lines as above with $Y$ defined using $\tau$ and $\alpha$, we obtain the uniqueness of the solution as a fixed point of $N$.

The result is also as follows. 
THEOREM 1. Let us consider the problem on $\mathbb{R}^{N}, N=1,2,3$,

$$
\left\{\begin{array}{l}
i \dot{\phi}+\Delta \phi=\nabla \Delta^{-1} \cdot(n \phi) \\
\ddot{n}-\Delta n=\Delta|\phi|^{2} \\
n(x, 0)=n_{0}(x) \\
\partial_{t} n(x, 0)=n_{1}(x) \\
\phi(x, 0)=\phi_{0}(x)
\end{array}\right.
$$

with $n_{0} \in H^{1}, n_{1} \in L^{2}$ and $\phi_{0} \in H^{2}$.

Then there exists a time $T>0$ depending only on $\left\|n_{0}\right\|_{H^{1}},\left\|n_{1}\right\|_{L^{2}},\left\|\phi_{0}\right\|_{H^{2}}$ and $N$ and a unique solution $(\phi(t), n(t))$ with

$$
\left\{\begin{array}{l}
\phi(t) \in \mathcal{C}^{0}\left([0, T] ; H^{2}\right) \cap \mathcal{C}^{1}\left([0, T] ; L^{2}\right) \\
\phi(t) \in W^{1,8 / N}\left(0, T ; L^{4}\right) \\
n(t) \in \mathcal{C}^{0}\left([0, T] ; H^{1}\right) \cap \mathcal{C}^{1}\left([0, T] ; L^{2}\right) \cap \mathcal{C}^{2}\left([0, T] ; H^{-1}\right)
\end{array}\right.
$$

Remark. The former result is a result about the local Cauchy problem. Thanks to the two conservation laws (34) and (35) we may show, as for the classical Zakharov equation (see [6]), that the solutions are global in time in the 1-dimensional case as well as in the 2-dimensional case for sufficiently small initial data.

\section{LIMIT WHEN $\lambda \rightarrow \infty$}

We consider the problem

where $B=\nabla\left(\Delta^{-1}\right) \nabla$, and

$$
\left\{\begin{array}{l}
\frac{1}{\lambda^{2}} n_{t t}-\Delta\left(n+|E|^{2}\right)=0 \\
i E_{t}+\Delta E+B(n E)=0
\end{array}\right.
$$

$$
\left\{\begin{array}{l}
E: \mathbb{R}_{x}^{k} \times \mathbb{R}_{t}^{+} \rightarrow \mathbb{C}^{k}, \\
n: \mathbb{R}_{x}^{k} \times \mathbb{R}_{t}^{+} \rightarrow \mathbb{R} .
\end{array}\right.
$$

Our goal is to show that the solutions $(n, E)$ to (36) tend to $\left(-|\tilde{E}|^{2}, \tilde{E}\right)$ when $\lambda$ goes to infinity, where $\tilde{E}$ is the solution to $i \tilde{E}_{t}+\nabla \tilde{E}=B\left(|\tilde{E}|^{2} \tilde{E}\right)$. We follow the work of Schochet and Weinstein [7]. We have encountered some new difficulties due to the nonlocal term. This complicates the transformation of the initial system and makes useful the use of commutators. We first have to prove the existence of solutions in $H^{s}$ with $s>[k / 2]+3$ for an interval of time which may be very small but independent of $\lambda$. To do that we proceed in two steps: the first one consists in writing the system as the perturbation of a symmetric hyperbolic system and the second one in computing the solution to this equivalent system as the limit of a sequence.

\subsection{Transformation of the system}

We want to describe the system (36) as a dispersive perturbation of a symmetric hyperbolic system. 
We set

$$
\left\{\begin{array}{l}
V=-\frac{1}{\lambda} \Delta^{-1} \nabla n_{t} \\
Q=n+|E|^{2}
\end{array}\right.
$$

where

$$
\left\{\begin{array}{l}
V: \mathbb{R}_{x}^{k} \times \mathbb{R}_{t}^{+} \rightarrow \mathbb{R}^{k}, \\
Q: \mathbb{R}_{x}^{k} \times \mathbb{R}_{t}^{+} \rightarrow \mathbb{R} .
\end{array}\right.
$$

Hence, (36) formally reads

$$
\begin{aligned}
& \left\{\begin{array}{l}
Q_{t}+\lambda \nabla \cdot V-\left(|E|^{2}\right)_{t}=0 \\
V_{t}+\lambda \nabla Q=0, \\
i E_{t}+\Delta E-B\left(|E|^{2}\right)+B(Q E)=0 .
\end{array}\right. \\
& i E_{t}+\Delta E-B\left(|E|^{2} E\right)+B(Q E)=0, \\
& i^{t} E_{t} \bar{E}+{ }^{t} \Delta E \bar{E}-{ }^{t} B(|E|) \bar{E}+{ }^{t} B(Q E) \bar{E}=0, \\
& i\left(|E|^{2}\right)_{t}+{ }^{t} \Delta E \bar{E}-{ }^{t} \Delta \bar{E} E-{ }^{t} B\left(|E|^{2} E\right) \bar{E}+{ }^{t} B\left(|E|^{2} \bar{E}\right) E+{ }^{t} B(Q E) \bar{E}-{ }^{t} B(Q \bar{E}) E=0 .
\end{aligned}
$$

Moreover,

$$
i \partial_{j} E_{t}+\Delta \partial_{j} E-\partial_{j} B\left(|E|^{2} E\right)+\partial_{j} B(Q E)=0,
$$

where $\partial_{j}$ is the differentiation with respect to $x_{j}$.

This may be written in a condensed form

$$
i \nabla E_{t}+\Delta \nabla E-\nabla B\left(|E|^{2} E\right)+\nabla B(Q E)=0,
$$

where, for a vector ${ }^{\prime}\left(\Phi^{1}, \ldots, \Phi^{k}\right)$, we have

$$
\nabla \Phi={ }^{t}\left(\partial_{1} \Phi^{1}, \partial_{1} \Phi^{2}, \ldots, \partial_{1} \Phi^{k}, \partial_{2} \Phi^{1}, \ldots, \partial_{k} \Phi^{k}\right) .
$$

We will call these $k^{2}$-components vectors, 2-vectors.

We also want to write the 2 -vector $\nabla B \Phi$ in the form $A \nabla \Phi$ where $A$ is always an operator of order 0 .

As $\nabla \cdot \Phi=\sum_{j=1}^{k} \partial_{j} \Phi^{j}$, we have $\nabla \cdot \Phi=\Gamma \cdot \nabla \Phi$, where $\Gamma$ is the 2-vector

$$
{ }^{t}(1,0, \ldots, 0 ; 0,1, \ldots, 0 ; \ldots ; 0, \ldots, 0,1)
$$

and, hence, $A=\nabla \nabla\left(\Delta^{-1}\right) \Gamma$, (transforms a 2-vector into a 2-vector). We also have

$$
i \nabla E_{t}+\Delta \nabla E-A \nabla\left(|E|^{2} E\right)+A \nabla(Q E)=0 .
$$

Now

$$
\begin{aligned}
\nabla(Q E) & ={ }^{t}\left(\partial_{1}\left(Q E^{1}\right), \partial_{1}\left(Q E^{1}\right), \ldots, \partial_{k}\left(Q E^{k}\right)\right), \\
& =Q \nabla E+{ }^{t}\left(\partial_{1} Q E^{1}, \partial_{1} Q E^{2}, \ldots, \partial_{k} Q E^{k}\right), \\
& =Q \nabla E+\alpha(E) \nabla Q,
\end{aligned}
$$

where $\alpha(\Phi)$ is the matrix $\left(k^{2}-k\right)$ which has $\Phi$ on its "diagonal" and zeros everywhere else. 
Similarly, $\nabla\left(|E|^{2} E\right)=|E|^{2} \nabla E+\alpha(E) \nabla\left(|E|^{2}\right)$,

$$
\begin{aligned}
\alpha(E) \nabla\left(|E|^{2}\right)= & \sum_{j=1}^{k}{ }^{t}\left(\partial_{1} E^{j} \bar{E}^{j} E^{1}, \partial_{1} E^{j} \bar{E}^{j} E^{2}, \ldots, \partial_{k} E^{j} \bar{E}^{j} E^{k}\right) \\
& +\sum_{j=1}^{k}{ }^{t}\left(\partial_{1} \bar{E}^{j} E^{j} E^{1}, \partial_{1} \bar{E}^{j} E^{j} E^{2}, \ldots, \partial_{k} \bar{E}^{j} E^{j} E^{k}\right) .
\end{aligned}
$$

Then we have $\alpha(E) \nabla\left(|E|^{2}\right)=\mathbb{Q}_{1}(E) \nabla E+\mathbb{Q}_{2}(E) \nabla \bar{E}$, where $\mathfrak{Q}_{1}(E)$ (resp. $\mathfrak{Q}_{2}(E)$ ) is the block-diagonal matrix $\left(k^{2}-k^{2}\right)$ such that each block is equal to $E^{t} \bar{E}$ (resp. $E^{t} E$ ). Hence

$$
i \nabla E_{t}+\Delta \nabla E-A\left(|E|^{2} \nabla E+\mathfrak{Q}_{1}(E) \nabla E+\mathfrak{Q}_{2}(E) \nabla \bar{E}\right)+A(Q \nabla E+\alpha(E) \nabla Q)=0 .
$$

We now set

$$
\sqrt{2} E=F+i G \quad \text { and } \quad \sqrt{2} \nabla E=H+i L
$$

Hence, we have

$$
\left\{\begin{array}{l}
F, G: \mathbb{R}_{x}^{k} \times \mathbb{R}_{t}^{+} \rightarrow \mathbb{R}^{k}, \\
H, L: \mathbb{R}_{x}^{k} \times \mathbb{R}_{t}^{+} \rightarrow \mathbb{R}^{k^{2}} .
\end{array}\right.
$$

Moreover, we notice that $B$ and $A$ preserve the real and imaginary parts,

$$
\begin{gathered}
\left(|E|^{2}\right)_{t}=i\left({ }^{t} \Delta E \bar{E}-{ }^{t} \Delta \bar{E} E-{ }^{t} B\left(|E|^{2} E\right) \bar{E}+{ }^{t} B\left(|E|^{2} \bar{E}\right) E+{ }^{t} B(Q E) \bar{E}-{ }^{t} B(Q \bar{E}) E\right), \\
{ }^{t} \Delta E \cdot \bar{E}=\sum_{j=1}^{k}\left(\bar{E}^{j} \sum_{l=1}^{k} \partial_{l}\left(\partial_{l} E^{j}\right)\right)={ }^{t} \bar{E} \nabla \cdot(\nabla E),
\end{gathered}
$$

where $\nabla \cdot$ is the multiplication by the following $\left(k-k^{2}\right)$ matrix

$$
\begin{aligned}
& \left(\begin{array}{cccccccccc}
\partial_{1} & & 0 & \partial_{2} & & 0 & & \partial_{k} & & 0 \\
& \ddots & & & \ddots & & \ldots & & \ddots & \\
0 & & \partial_{1} & 0 & & \partial_{2} & & 0 & & \partial_{k}
\end{array}\right) \\
& \left(|E|^{2}\right)_{t}=i\left({ }^{t} \Delta E \bar{E}-{ }^{t} \Delta \bar{E} E-{ }^{t} B\left(|E|^{2} E\right) \bar{E}+{ }^{t} B\left(|E|^{2} \bar{E}\right) E+{ }^{t} B(Q E) \bar{E}-{ }^{t} B(Q \bar{E}) E\right) \text {, } \\
& =i\left(-i^{t} G \nabla \cdot H+i^{t} F \nabla \cdot L-i \frac{B}{4}\left(\left(|F|^{2}+|G|^{2}\right)(F+i G)\right)(F-i G)\right. \\
& +i \frac{B}{4}\left(\left(|F|^{2}+|G|^{2}\right)(F-i G)\right)(F+i G) \\
& \left.+i^{t} B(Q(F+i G))(F-i G)-i^{t} B(Q(F-i G))(F+i G)\right) \\
& =\left({ }^{t} G \nabla \cdot H-{ }^{t} F \nabla \cdot L\right)+\left(-\frac{{ }^{t} B}{2}\left(\left(|F|^{2}+|G|^{2}\right) F\right) G+\frac{{ }^{t} B}{2}\left(\left(|F|^{2}+|G|^{2}\right) G\right) F\right) \\
& +\left({ }^{t} B(Q F) G-{ }^{t} B(Q G) F\right) \text {. }
\end{aligned}
$$




$$
\begin{gathered}
Q_{t}+\lambda \nabla \cdot V+{ }^{t} F \nabla \cdot L-{ }^{t} G \nabla \cdot H+\frac{{ }^{t} B}{2}\left(\left(|F|^{2}+|G|^{2}\right) F\right) G \\
-\frac{{ }^{t} B}{2}\left(\left(|F|^{2}+|G|^{2}\right) G\right) F-{ }^{t} B(Q F) G+{ }^{t} B(Q G) F=0 \\
V_{t}+\lambda \nabla Q=0 \\
i(F+i G)_{t}+\Delta(F+i G)-\frac{1}{2} B\left(\left(|F|^{2}+|G|^{2}\right)(F+i G)\right)+B(Q(F+i G))=0 \\
F_{t}+\Delta G-\frac{1}{2} B\left(\left(|F|^{2}+|G|^{2}\right) G\right)+B(Q G)=0 \\
G_{t}-\Delta F+\frac{1}{2} B\left(\left(|F|^{2}+|G|^{2}\right) F\right)-B(Q F)=0 \\
i(H+i L)_{t}+\Delta(H+i L) \\
-\frac{A}{2}\left(\left(|F|^{2}+|G|^{2}\right)(H+i L)+\mathbb{Q}_{1}(F+i G)(H+i L)+Q_{2}(F+i G)(H-i L)\right) \\
+A(Q(H+i L)+\alpha(F+i G) \nabla Q)=0
\end{gathered}
$$

In order to treat $Q_{1}$ and $Q_{2}$, we have

$$
\begin{aligned}
& E^{t} \bar{E}=(F+i G)^{t}(F-i G)=F^{t} F+G^{t} G+i\left(G^{t} F-F^{t} G\right), \\
& E^{t} E=(F+i G)^{t}(F+i G)=F^{t} F-G^{t} G+i\left(G^{t} F+F^{t} G\right),
\end{aligned}
$$

Let $\mathfrak{N}$ be the operator which creates a $\left(k^{2}-k^{2}\right)$-dimensional block-diagonal matrix with identical blocks. Then

$$
\begin{aligned}
& \mathfrak{Q}_{1}(F+i G)(H+i L)+\mathfrak{Q}_{2}(F+i G)(H-i L) \\
& =\mathfrak{T}\left(F^{t} F+G^{t} G\right) H-\mathfrak{T}\left(G^{t} F-F^{t} G\right) L+\mathfrak{K}\left(F^{t} F-G^{t} G\right) H+\mathfrak{K}\left(G^{t} F+F^{t} G\right) L \\
& +i\left[\mathfrak{T}\left(F^{t} F+G^{t} G\right) L+\mathfrak{K}\left(G^{t} F-F^{t} G\right) H-\mathfrak{N}\left(F^{t} F-G^{t} G\right) L+\mathfrak{N}\left(G^{t} F+F^{t} G\right) H\right] \\
& =2 \mathfrak{K}\left(F^{t} F\right) H+2 \mathfrak{N}\left(F^{t} G\right) L+2 i\left[\mathfrak{K}\left(G^{t} G\right) L+\mathfrak{N}\left(G^{t} F\right) H\right] \text {, } \\
& H_{t}+\Delta L-A\left(\frac{1}{2}\left(|F|^{2}+|G|^{2}\right) L+\mathfrak{K}\left(G^{t} G\right) L+\mathfrak{T}\left(G^{t} F\right) H\right)+A(Q L+\alpha(G) \nabla Q)=0, \\
& L_{t}-\Delta H+A\left(\frac{1}{2}\left(|F|^{2}+|G|^{2}\right) H+\mathfrak{N}\left(F^{t} F\right) H+\mathfrak{K}\left(F^{t} G\right) L\right)-A(Q H+\alpha(F) \nabla Q)=0 .
\end{aligned}
$$

Summing up, we have the following system

$$
\begin{aligned}
Q_{t}+ & \lambda \nabla \cdot V+{ }^{t} F \nabla \cdot L-{ }^{t} G \nabla \cdot H+\frac{{ }^{t} B}{2}\left(\left(|F|^{2}+|G|^{2}\right) F\right) G \\
& -\frac{{ }^{t} B}{2}\left(\left(|F|^{2}+|G|^{2}\right) G\right) F-{ }^{t} B(Q F) G+{ }^{t} B(Q G) F=0,
\end{aligned}
$$




$$
\begin{gathered}
V_{t}+\lambda \nabla Q=0, \\
F_{t}+\Delta G-\frac{1}{2} B\left(\left(|F|^{2}+|G|^{2}\right) G\right)+B(Q G)=0, \\
G_{t}-\Delta F+\frac{1}{2} B\left(\left(|F|^{2}+|G|^{2}\right) F\right)-B(Q F)=0, \\
H_{t}+\Delta L-A\left(\frac{1}{2}\left(|F|^{2}+|G|^{2}\right) L+\mathfrak{K}\left(G^{t} G\right) L+\mathfrak{K}\left(G^{t} F\right) H\right)+A(Q L+\alpha(G) \nabla Q)=0, \\
\left.L_{t}-\Delta H+A\left(\frac{1}{2}\left(|F|^{2}+|G|^{2}\right) H+\mathfrak{M}\left(F^{t} F\right) H+\mathfrak{T}\left(F^{t} G\right) L\right)-A(Q H)+\alpha(F) \nabla Q\right)=0,
\end{gathered}
$$

which also reads

$$
\begin{gathered}
Q_{t}+\lambda \nabla \cdot V+{ }^{t} F \nabla \cdot L-{ }^{t} G \nabla \cdot H+\frac{{ }^{t} B}{2}\left(\left(|F|^{2}+|G|^{2}\right) F\right) G \\
-\frac{{ }^{t} B}{2}\left(\left(|F|^{2}+|G|^{2}\right) G\right) F-{ }^{t} B(Q F) G+{ }^{t} B(Q G) F=0, \\
V_{t}+\lambda \nabla Q=0, \\
F_{t}-\frac{1}{2} B\left(\left(|F|^{2}+|G|^{2}\right) G\right)+B(Q G)=-\Delta G \\
G_{t}+\frac{1}{2} B\left(\left(|F|^{2}+|G|^{2}\right) F\right)-B(Q F)=\Delta F \\
H_{t}-A\left(\frac{1}{2}\left(|F|^{2}+|G|^{2}\right) L+\mathfrak{N}\left(G^{t} G\right) L+\mathfrak{T}\left(G^{t} F\right) H\right)+A(Q L+\alpha(G) \nabla Q)=-\Delta L, \\
L_{t}+A\left(\frac{1}{2}\left(|F|^{2}+|G|^{2}\right) H+\mathfrak{N}\left(F^{t} F\right) H+\mathfrak{N}\left(F^{t} G\right) L\right)-A(Q H+\alpha(F) \nabla Q)=\Delta H
\end{gathered}
$$

We introduce the vector with $1+3 k+2 k^{2}$ components: $U={ }^{t}(Q, V, F, G, H, L)$, and we want to write the above system in the form

$$
U_{t}+\sum_{j=1}^{k}\left\{R\left(A^{j}(U) U_{x j}\right)+\lambda C^{j} U_{x j}\right\}+S(\tilde{B}(U) U)=K \Delta U
$$

where $R$ and $S$ are nonlocal operators.

Let us describe all the operators

$$
K=\left(\begin{array}{rrrrrr}
0 & 0 & 0 & 0 & 0 & 0 \\
0 & 0 & 0 & 0 & 0 & 0 \\
0 & 0 & 0 & -I & 0 & 0 \\
0 & 0 & I & 0 & 0 & 0 \\
0 & 0 & 0 & 0 & 0 & -I \\
0 & 0 & 0 & 0 & I & 0
\end{array}\right)
$$




$$
C^{j}=\left(\begin{array}{cccccc}
0 & { }^{i} \varepsilon^{j} & 0 & 0 & 0 & 0 \\
\varepsilon^{j} & 0 & 0 & 0 & 0 & 0 \\
0 & 0 & 0 & 0 & 0 & 0 \\
0 & 0 & 0 & 0 & 0 & 0 \\
0 & 0 & 0 & 0 & 0 & 0 \\
0 & 0 & 0 & 0 & 0 & 0
\end{array}\right),
$$

where $\varepsilon^{j}$ is the $j$ th vector of the canonical basis of $\mathbb{R}^{k}$.

$$
A^{j}(U)=\left(\begin{array}{ccccccc}
0 & 0 & 0 & 0 & -{ }^{t}\left(\alpha(G) \varepsilon^{j}\right) & { }^{t}\left(\alpha(F) \varepsilon^{j}\right) \\
0 & 0 & 0 & 0 & 0 & 0 \\
0 & 0 & 0 & 0 & 0 & 0 \\
0 & 0 & 0 & 0 & 0 & 0 \\
-\alpha(G) \varepsilon^{j} & 0 & 0 & 0 & 0 & 0 \\
\alpha(F) \varepsilon^{j} & 0 & 0 & 0 & & 0 & 0 \\
R & =\left(\begin{array}{cccccc}
I & 0 & 0 & 0 & 0 & 0 \\
0 & I & 0 & 0 & 0 & 0 \\
0 & 0 & I & 0 & 0 & 0 \\
0 & 0 & 0 & I & 0 & 0 \\
0 & 0 & 0 & 0 & -A & 0 \\
0 & 0 & 0 & 0 & 0 & -A
\end{array}\right) .
\end{array}\right.
$$

We denote by $\tilde{B}_{j}(U)$ the $j$ th column of $\tilde{B}_{j}$, and we have

$$
\tilde{B}_{3}(U)=\left(\begin{array}{c}
\tilde{B}_{1}(U)=\tilde{B}_{2}(U) \equiv 0, \\
-\frac{1}{2} t^{t} B\left(\left(|F|^{2}+|G|^{2}\right) G\right)+{ }^{t} B(Q F) \\
0 \\
0 \\
-\frac{1}{2}\left(|F|^{2}+|G|^{2}\right)+Q \\
0 \\
0
\end{array}\right)
$$




$$
\begin{aligned}
& \tilde{B}_{4}(U)=\left(\begin{array}{c}
\frac{1}{2}^{t} B\left(\left(|F|^{2}+|G|^{2}\right) F\right)-{ }^{t} B(Q G) \\
0 \\
\frac{1}{2}\left(|F|^{2}+|G|^{2}\right)-Q \\
0 \\
0 \\
0
\end{array}\right) \\
& \tilde{B}_{5}(U)=\left(\begin{array}{c}
0 \\
0 \\
0 \\
0 \\
\mathfrak{M}\left(G^{t} F\right) \\
-\frac{1}{2}\left(|F|^{2}+|G|^{2}\right)-\mathfrak{M}\left(F^{t} F\right)+Q
\end{array}\right) \\
& \tilde{B}_{6}(U)=\left(\begin{array}{c}
0 \\
0 \\
0 \\
0 \\
\frac{1}{2}\left(|F|^{2}+|G|^{2}\right)+\mathfrak{N}\left(G^{t} G\right)-Q \\
-\mathfrak{N}\left(F^{t} G\right)
\end{array}\right)
\end{aligned}
$$

$\tilde{B}(U)$ has a nonlocal term in its first component

$$
S=\left(\begin{array}{rrrrrr}
I & 0 & 0 & 0 & 0 & 0 \\
0 & I & 0 & 0 & 0 & 0 \\
0 & 0 & -B & 0 & 0 & 0 \\
0 & 0 & 0 & -B & 0 & 0 \\
0 & 0 & 0 & 0 & -A & 0 \\
0 & 0 & 0 & 0 & 0 & -A
\end{array}\right)
$$

\subsection{Existence of a regular solution for an independent time of $\lambda$}

We set $N=2 k^{2}+3 k+1$. We consider the following iteration scheme

$$
\begin{gathered}
U^{0}(x, t)=U_{0}(x), \\
\frac{\partial U^{p+1}}{\partial t}+\sum_{j=1}^{k}\left\{R\left(A^{j}\left(U^{p}\right) U_{x_{j}}^{p+1}\right)+\lambda C^{j} U_{x_{j}}^{p+1}\right\}+S\left(\tilde{B}\left(U^{p}\right) U^{p+1}\right)=K \Delta U^{p+1}, \\
U^{p+1}(x, 0)=U_{0}(x) .
\end{gathered}
$$


We set

$$
\|u\|_{s}=\|u\|_{s, T}=\sup _{t \in[0, T]}\|u(\cdot, t)\|_{s}
$$

In what follows, we assume that $s>[k / 2]+3$.

Moreover, we set $\left\|U_{0}\right\|_{s}=\varepsilon$ and let $\delta>\varepsilon$. We argue as follows

- $\forall p \geq 0\left\|U^{p}\right\|_{s, T} \leq \delta$.

- $\forall p \geq 0\left\|U^{p+1}-U^{p}\right\|_{0, T} \leq C\left\|U^{p}-U^{p-1}\right\|_{0, T}$ with $C<1$.

- Then

$$
U^{p} \rightarrow U \quad \text { in } L^{\infty}\left(0, T ; L^{2}\right),
$$

$U^{p}$ is bounded in $L^{\infty}\left(0, T ; H^{s}\right)$

Thanks to the convexity of the \|\|$_{s}$ norms, we have

$$
\left\|\left(U^{p}-U\right)(\cdot, t)\right\|_{s^{\prime}} \rightarrow 0 \quad \forall s^{\prime}<s .
$$

As $s>k / 2+1$, we may choose $s^{\prime}>k / 2+1$. Then $U \in \mathcal{C}\left([0, T] ; \mathfrak{C}^{\mathbf{l}}\right)$ and the solution is a classical one. Moreover, we have

$$
\begin{gathered}
U \in \operatorname{Lip}\left([0, T] ; H^{s-2}\right) \\
U \in \mathcal{C}\left([0, T] ; H^{s}\right) \cap \mathcal{C}^{1}\left([0, T] ; H^{s-2}\right) .
\end{gathered}
$$

There remains to specify the first two points.

4.2.1. A lemma. In what follows, we use the following result. Let us consider the problem

$$
\partial_{t} u+a(u)=f
$$

with $a(u)=\sum_{j=1}^{k}\left\{R\left(A^{j}(v) \partial_{j} u\right)+\lambda C^{j} \partial_{j} u\right\}+S(\tilde{B}(v) u)-K \Delta u$, where $\|v\|_{s, T} \leq \delta$,

$$
\left(\left(\partial_{t} u, u\right)\right)+((a(u), u)) \leq\|f\|_{0}\|u\|_{0} .
$$

$C^{j}$ is symmetric with constant coefficients, so we have $\left(\left(\lambda C^{j} \partial_{j} u, u\right)\right)=0$. This leads to a result which does not depend on $\lambda$. $K$ being antisymmetric, $((K \Delta u, u))=0$. Hence,

$$
\begin{gathered}
((a(u), u))=\sum_{j=1}^{k}\left(\left(R\left(A^{j}(v) \partial_{j} u\right), u\right)\right)+((S(\tilde{B}(v) u), u)), \\
|((S(\tilde{B}(v) u), u))| \leq\|S(\tilde{B}(v) u)\|_{0}\|u\|_{0} \leq C\|u\|_{0}^{2},
\end{gathered}
$$

where $C$ only depends on $\delta$. 
There also remains to estimate $\left(\left(R\left(A^{j}(v) \partial_{j} u\right), u\right)\right)$ for all $0 \leq j \leq k$,

$$
\begin{aligned}
\left(\left(R\left(A^{j}(v) \partial_{j} u\right), u\right)\right) & =\left(\left(R\left(\partial_{j}\left(A^{j}(v) u\right)\right), u\right)\right)-(\underbrace{\left(\left(R\left(\left[A^{j}(v), \partial_{j}\right] u\right), u\right)\right.}_{\alpha}) \\
& =\left(\left(\partial_{j} R\left(A^{j}(v) u\right), u\right)\right)-\alpha \\
& =-\left(\left(R\left(A^{j}(v) u\right), \partial_{j} u\right)\right)-\alpha \\
& =-\left(\left(A^{j}(v) u, R\left(\partial_{j} u\right)\right)\right)-\alpha \\
& =-\left(\left(u, A^{j}(v) R\left(\partial_{j} u\right)\right)\right)-\alpha \\
& =-\left(\left(u, R\left(A^{j}(v) \partial_{j} u\right)\right)\right)-\alpha-\left(\left(\left[A^{j}(v), R\right]\left(\partial_{j} u\right), u\right)\right) \\
2\left(\left(R\left(A^{j}(v) \partial_{j} u\right), u\right)\right) & =-\left(\left(R\left(\left[A^{j}(v), \partial_{j}\right] u\right), u\right)\right)-\left(\left(\left[A^{j}(v), R\right]\left(\partial_{j} u\right), u\right)\right) .
\end{aligned}
$$

Hence $2\left(\left(R\left(A^{j}(v) \partial_{j} u\right), u\right)\right)=\left(\left(R\left(\left[\partial_{j}, A^{j}(v)\right] u\right), u\right)\right)+\left(\left(\left[R, A^{j}(v)\right]\left(\partial_{j} u\right), u\right)\right) . \quad\left[\partial_{j}, A^{j}(v)\right] \quad$ is $\quad$ a commutator of order 0 , hence

$$
\left|\left(\left(R\left(\left[\partial_{j}, A^{j}(v)\right] u\right), u\right)\right)\right| \leq C\|u\|_{0}^{2},
$$

and the constant only depends on $\delta .\left[R, A^{j}(v)\right]$ is a commutator of order -1 , therefore,

$$
\left.\mid\left(\left(\left[R, A^{j}(v)\right] \partial_{j} u\right), u\right)\right) \mid \leq C\|u\|_{0}^{2},
$$

and the constant only depends on $\delta$.

Also, we have

$$
\frac{\mathrm{d}}{\mathrm{d} t}\|u(t)\|_{0}^{2} \leq C\|u(t)\|_{0}^{2}+2\|f\|_{0}\|u\|_{0},
$$

where $C$ only depends on $\delta$.

4.2.2. Estimation for the large norm. We are going to show the result by induction: we consider the following assumption.

$\left(\mathrm{H}_{p}\right) \forall 0 \leq l \leq p\left\|U^{l}\right\|_{s, T} \leq \delta$.

This is valid for $p=0$ (because $\varepsilon \leq \delta$ ).

We want to show that $\left(H_{p}\right) \Rightarrow\left(H_{p+1}\right)$ (for a good choice of $T$ ). We choose $\alpha$ such that $|\alpha| \leq s$, and we set $U_{\alpha}^{p+1}=D^{\alpha} U^{p+1}$.

$$
\left\{\begin{array}{c}
\partial_{t} U_{\alpha}^{p+1}+\sum_{j=1}^{k}\left\{R\left(A^{j}\left(U^{p}\right) \partial_{j} U_{\alpha}^{p+1}\right)+\lambda C^{j} \partial_{j} U_{\alpha}^{p+1}\right\}+S\left(\tilde{B}\left(U^{p}\right) U_{\alpha}^{p+1}\right)-K \Delta U_{\alpha}^{p+1} \\
=\left(\sum_{j=1}^{k}\left[R A^{j}\left(U^{p}\right) \partial_{j}, D^{\alpha}\right]+\left[S \tilde{B}\left(U^{p}\right), D^{\alpha}\right]\right) U^{p+1} .
\end{array}\right.
$$

The commutator $\sum_{j=1}^{k}\left[R A^{j}\left(U^{p}\right) \partial_{j}, D^{\alpha}\right]+\left[S \tilde{B}\left(U^{p}\right), D^{\alpha}\right]$ is an operator of order $|\alpha|$.

This equation is in the form

$$
\partial_{t} U_{\alpha}^{p+1}+a\left(U_{\alpha}^{p+1}\right)=f_{1},
$$


with $v=U^{p}$ (and hence $\|v\|_{s, T} \leq \delta$ ), and $f_{1}=\left[\sum_{j=1}^{k} R A^{j}\left(U^{p}\right) \partial_{j}+S \tilde{B}\left(U^{p}\right), D^{\alpha}\right] U^{p+1}$,

$$
\left\|f_{1}\right\|_{0} \leq C\left\|U^{p+1}\right\|_{|\alpha|} \leq C\left\|U^{p+1}\right\|_{s},
$$

and $C$ only depends on $\delta$. Using $(*)$, we obtain

$$
\frac{\mathrm{d}}{\mathrm{d} t}\left\|U_{\alpha}^{p+1}(t)\right\|_{0}^{2} \leq C\left(\left\|U_{\alpha}^{p+1}(t)\right\|_{0}^{2}+\left\|U^{p+1}\right\|_{s}\left\|U_{\alpha}^{p+1}\right\|_{0}\right)
$$

Hence,

$$
\begin{gathered}
\frac{\mathrm{d}}{\mathrm{d} t}\left\|U^{p+1}(t)\right\|_{s}^{2} \leq C\left\|U^{p+1}(t)\right\|_{s}^{2}, \\
\left\|U^{p+1}(t)\right\|_{s}^{2} \leq \mathrm{e}^{C t}\left\|U^{p+1}(0)\right\|_{s}^{2} \leq \mathrm{e}^{C t} \varepsilon^{2},
\end{gathered}
$$

and $C$ only depends on $\delta$.

We choose $T$ sufficiently small such that

$$
\left\|U^{p+1}(t)\right\|_{s} \leq \delta \quad \forall 0 \leq t \leq T
$$

$T$ does not depend on $p$ (nor on $\lambda$ ) because $C$ does not depend on them. Therefore, we have the estimation for the large norm.

\subsubsection{Convergence in low norm.}

$$
\begin{gathered}
\partial_{t} U^{p+1}+\sum_{j=1}^{k}\left\{R\left(A^{j}\left(U^{p}\right) U_{x_{j}}^{p+1}\right)+\lambda C^{j} U_{x_{j}}^{p+1}\right\}+S\left(\tilde{B}\left(U^{p}\right) U^{p+1}\right)-K \Delta U^{p+1}=0 \\
\partial_{t} U^{p}+\sum_{j=1}^{k}\left\{R\left(A^{j}\left(U^{p-1}\right) U_{x_{j}}^{p}\right)+\lambda C^{j} U_{x_{j}}^{p}\right\}+S\left(\tilde{B}\left(U^{p-1}\right) U^{p}\right)-K \Delta U^{p}=0 .
\end{gathered}
$$

Setting $V^{p}=U^{p+1}-U^{p}$, we obtain

$$
\left\{\begin{array}{l}
\partial_{t} V^{p}+\sum_{j=1}^{k}\left\{R\left(A^{j}\left(U^{p}\right) V_{x_{j}}^{p}\right)+\lambda C^{j} V_{x_{j}}^{p}\right\}+S\left(\tilde{B}\left(U^{p}\right) V^{p}\right)-K \Delta V^{p} \\
\quad=-\sum_{j=1}^{k} R\left(\left(A^{j}\left(U^{p}\right)-A^{j}\left(U^{p-1}\right)\right) U_{x_{j}}^{p}\right)-S\left(\left(\tilde{B}\left(U^{p}\right)-\tilde{B}\left(U^{p-1}\right)\right) U^{p}\right) \\
V^{p}(0, x)=0 .
\end{array}\right.
$$

This equation is in the form

$$
\partial_{j} V^{p}+a\left(V^{p}\right)=f_{2},
$$

with $v=U^{p}$, and $f_{2}=-\sum_{j=1}^{k} R\left(\left(A^{j}\left(U^{p}\right)-A^{j}\left(U^{p-1}\right)\right) U_{x_{i}}^{p}\right)-S\left(\left(\tilde{B}\left(U^{p}\right)-\tilde{B}\left(U^{p-1}\right)\right) U^{p}\right)$. 
$A^{j}$ is linear and $\tilde{B}$ only consists in terms of the first, second and third order, we have also

$$
\begin{aligned}
\left\|R\left(\left(A^{j}\left(U^{p}\right)-A^{j}\left(U^{p-1}\right)\right) U_{x_{j}}^{p}\right)\right\|_{0} & \leq C\left\|U^{p}-U^{p-1}\right\|_{0}\left\|U_{x_{j}}^{p}\right\|_{\infty} \\
& \leq C\left\|U^{p}-U^{p-1}\right\|_{0}\left\|U^{p}\right\|_{s} \\
& \leq C\left\|U^{p}-U^{p-1}\right\|_{0}, \\
\left\|S\left(\left(\tilde{B}\left(U^{p}\right)-\tilde{B}\left(U^{p-1}\right)\right) U^{p}\right)\right\|_{0} & \leq C\left\|U^{p}-U^{p-1}\right\|_{0}\left\|U^{p}\right\|_{\infty} \\
& \leq C\left\|U^{p}-U^{p-1}\right\|_{0}\left\|U^{p}\right\|_{s} \\
& \leq C\left\|U^{p}-U^{p-1}\right\|_{0},
\end{aligned}
$$

where the constants only depend on $\delta$.

Using (*), we have

$$
\frac{\mathrm{d}}{\mathrm{d} t}\left\|V^{p}(t)\right\|_{0}^{2} \leq C\left\|V^{p}\right\|_{0}^{2}+D\left\|V^{p-1}\right\|_{0}\left\|V^{p}\right\|_{0}
$$

Hence

$$
\frac{\mathrm{d}}{\mathrm{d} t}\left\|V^{p}(t)\right\|_{0}^{2} \leq C\left\|V^{p}\right\|_{0}^{2}+\beta\left\|V^{p-1}\right\|_{0}^{2}+\frac{D^{2}}{4 \beta}\left\|V^{p}\right\|_{0}^{2} .
$$

We consider $\beta$ such that $C+D^{2} / 4 \beta=\beta\left(4 \beta^{2}-C \beta-D^{2}=0\right.$ admits a positive solution $\left.\beta\right)$

$$
\frac{\mathrm{d}}{\mathrm{d} t}\left\|V^{p}(t)\right\|_{0}^{2} \leq \beta\left\|V^{p}\right\|_{0}^{2}+\beta\left\|V^{p-1}\right\|_{0}^{2}
$$

then

$$
\left\|V^{p}(t)\right\|_{0}^{2} \leq \mathrm{e}^{\beta t} \underbrace{\left\|V^{p}(0)\right\|_{0}^{2}}_{0}+\left(\mathrm{e}^{\beta t}-1\right)\left\|V^{p-1}(t)\right\|_{0}^{2} .
$$

We choose $T$ sufficiently small such that $\mathrm{e}^{\beta t}-1<1 \forall 0 \leq t \leq T$. Hence we obtain the convergence in the low norm.

4.2.4. Return to the initial problem. We suppose that the Cauchy problem is posed for the following initial data

$$
\left\{\begin{array}{l}
n(0, x)=n_{0}(x) \\
\partial_{t} n(0, x)=n_{1}(x), \\
E(0, x)=E_{0}(x)
\end{array}\right.
$$

$V_{t}+\lambda \nabla Q=0$ is one of the given equations. $F_{t}-\frac{1}{2} B\left(\left(|F|^{2}+|G|^{2}\right) G\right)+B(Q G)=-\Delta G$, and $G_{t}+\frac{1}{2} B\left(\left(|F|^{2}+|G|^{2}\right) F\right)-B(Q F)=\Delta F$ immediately yields

$$
E_{t}+\Delta E-B\left(|E|^{2} E\right)+B(Q E)=0,
$$

where we have set $E=1 / \sqrt{2}(F+i G)$. 
Let us set $W=(\nabla F-H, \nabla G-L)$, this is zero at time $t=0$ and, therefore, for all $t$, hence $\nabla E=1 / \sqrt{2}(H+i L)$ and using the last equations of the perturbated hyperbolic system we have $Q_{t}+\lambda \nabla \cdot V-\left(|E|^{2}\right)_{t}=0$. Therefore, we find again the initial equations.

We can also get some regularity

$$
\begin{gathered}
E \in \mathcal{C}\left([0, T] ; H^{s+1}\right) \cap \mathfrak{C}^{1}\left([0, T] ; H^{s-1}\right), \\
n \in \mathcal{C}\left([0, T] ; H^{s}\right) \cap \mathcal{C}^{1}\left([0, T] ; H^{s-1}\right) \cap \mathcal{C}^{2}\left([0, T] ; H^{s-2}\right) .
\end{gathered}
$$

Hence

$$
\left\{\begin{array}{l}
\frac{1}{\lambda^{2}} n_{t t}-\Delta\left(n+|E|^{2}\right)=0, \\
i E_{t}+\Delta E+B(n E)=0,
\end{array}\right.
$$

and the solutions are classical ones.

In what follows, we call $\left(n^{\lambda}, E^{\lambda}\right)$, the solutions to the initial system (36) which is associated to the parameter $\lambda$. Then we have the following estimation

$$
\left\|E^{\lambda}\right\|_{H^{s+1}}+\left\|E_{t}^{\lambda}\right\|_{H^{s-1}}+\left\|n^{\lambda}\right\|_{H^{s}}+\frac{1}{\lambda}\left\|n_{t}^{\lambda}\right\|_{H^{s-1}}+\frac{1}{\lambda^{2}}\left\|n_{t t}^{\lambda}\right\|_{H^{s-2}} \leq \text { const. }
$$

We may state the following theorem.

THEOREM 2. Let $s \geq[k / 2]+3$. There exists a unique solution to (36), (45) on a time interval $[0, T], T$ not depending on $\lambda$ but only on $\left\|n_{0}\right\|_{H^{s}},\left\|n_{1}\right\|_{H^{s-1}}$ and $\left\|E_{0}\right\|_{H^{s+1}}$, which we suppose are finite.

Moreover, for all $t \in[0, T]$ we have the estimation (46).

\subsection{The limit when $\lambda$ tends to $\infty$}

TheOREM 3. When $\lambda$ tends to $\infty, n^{\lambda}+\left|E^{\lambda}\right|^{2} \rightarrow 0$ in $\mathcal{C}^{0}\left([0, T] \times \mathbb{R}^{k}\right), \nabla\left(n^{\lambda}+\left|E^{\lambda}\right|^{2}\right) \rightarrow 0$ in $\mathcal{C}^{0}\left([0, T] ; H^{s-2}\right), E^{\lambda} \rightarrow \tilde{E}$ in $\mathcal{C}^{1}\left([0, T] \times \mathbb{R}^{k}\right) \cap \mathcal{C}\left([0, T] ; \mathcal{C}^{2}\right)$, where $\tilde{E}$ is the unique solution to

$$
i \tilde{E}_{t}+\Delta \tilde{E}-B\left(|\tilde{E}|^{2} \tilde{E}\right)=0 \text {. }
$$

The proof is carried out as follows. We differentiate with respect to the time the equation (44), and obtain

$$
U_{t t}+\sum_{j=1}^{k}\left\{R\left(A^{j}(U) U_{x_{j} t}+A^{j}\left(U_{t}\right) U_{x_{j}}\right)+\lambda C^{j} U_{x_{j} t}\right\}+S\left(\tilde{B}_{t}(U) U+\tilde{B}(U) U_{t}\right)=K \Delta U_{t} .
$$

As in the former proof, we may then show that

$$
\left\|U_{t}^{\lambda}(0)\right\|_{H^{s-2}} \leq C \Rightarrow\left\|U_{t}^{\lambda}(t)\right\|_{H^{s-2}} \leq C^{\prime} \quad \forall t \in[0, T] .
$$

Indeed, let $\alpha$ be such that $|\alpha| \leq s-2$,

$$
\begin{aligned}
& \partial_{t}\left(U_{t \alpha}\right)+\sum_{j=1}^{k}\left\{R\left(A^{j}(U)\left(U_{t \alpha}\right)_{x_{j}}\right)+\lambda C^{j}\left(U_{t \alpha}\right)_{x_{j}}\right\}+\tilde{B}(U) U_{t \alpha}-K \Delta U_{t \alpha} \\
& =D^{\alpha}\left(-\sum_{j=1}^{k} R\left(A^{j}\left(U_{t}\right) U_{x_{j}}\right)+S\left(\tilde{B}_{t}(U) U\right)\right)+\left(\sum_{j=1}^{k}\left[R A_{j}(U) \partial_{j}, D^{\alpha}\right]+\left[S \tilde{B}(U), D^{\alpha}\right]\right) U_{t} .
\end{aligned}
$$


We set

$$
\begin{aligned}
& f_{3}=D^{\alpha}\left(-\sum_{j=1}^{k} R\left(A^{j}\left(U_{t}\right) U_{x_{j}}\right)-S\left(\tilde{B}_{t}(U) U\right)\right) \\
& f_{4}=\left(\sum_{j=1}^{k}\left[R A_{j}(U) \partial_{j}, D^{\alpha}\right]+\left[S \tilde{B}(U), D^{\alpha}\right]\right) U_{t} \\
&\left\|f_{4}\right\|_{0} \leq C\left\|U_{t}\right\|_{s-2}, \\
&\left\|f_{3}\right\|_{0} \leq\left\|\sum_{j=1}^{k} R\left(A^{j}\left(U_{t}\right) U_{x_{j}}\right)+S\left(\tilde{B}_{t}(U) U\right)\right\|_{s-2} \\
&\left.\leq C \sum_{j=1}^{k}\left\|A^{j}\left(U_{t}\right) U_{x_{j}}\right\|_{s-2}+C \| \tilde{B}_{t}(U) U\right) \|_{s-2} \\
& \leq C\left\|U_{t}\right\|_{s-2} .
\end{aligned}
$$

We have an equation in the form

$$
\partial\left(U_{t \alpha}\right)+a\left(U_{t \alpha}\right)=f_{3}+f_{4},
$$

with $v=U$ and $\left\|f_{3}+f_{4}\right\|_{0} \leq C\left\|U_{t}\right\|_{s-2} \cdot(*)$ yields

$$
\begin{gathered}
\frac{\mathrm{d}}{\mathrm{d} t}\left\|U_{t \alpha}(t)\right\|_{0}^{2} \leq\left\|U_{t \alpha}(t)\right\|_{0}^{2}, \\
\left\|U_{t}(t)\right\|_{s-2}^{2} \leq \mathrm{e}^{C t}\left\|U_{t}(0)\right\|_{s-2}^{2},
\end{gathered}
$$

and $\left\|U_{t}(0)\right\|_{s-2}^{2}$ is finite using the initial regularity and equations (38)-(43). $V_{t}+\lambda \nabla Q=0$, then $V_{t}^{\lambda} \in L^{\infty}\left([0, T] ; H^{s-2}\right) \Rightarrow \lambda \nabla Q^{\lambda} \in L^{\infty}\left([0, T] ; H^{s-2}\right)$,

$$
\begin{aligned}
\Rightarrow\left\|Q^{\lambda}\right\|_{\mathfrak{e}^{0}\left([0, T] \times \mathbb{R}^{k}\right)} & \leq C\left\|D^{s-1} Q^{\lambda}\right\|_{L^{2}}^{k / 2(s-1)}\left\|Q^{\lambda}\right\|_{L^{2}}^{1-k / 2(s-1)} \\
& \leq C \lambda^{-k / 2(s-1)}
\end{aligned}
$$

and

$$
\left\|\nabla Q^{\lambda}\right\|_{\mathfrak{C}^{0}\left([0, T] ; H^{s-2}\right)} \leq C \lambda^{-1} .
$$

When $\lambda$ tends to $+\infty,\left\|Q^{\lambda}\right\|_{\mathfrak{C}^{0}\left([0, T] \times R^{k}\right)} \rightarrow 0$ and $\left\|\nabla Q^{\lambda}\right\|_{\mathcal{C}^{0}\left([0, T] ; H^{s-2}\right)} \rightarrow 0$, hence $n^{\lambda}+\left|E^{\lambda}\right|^{2} \rightarrow 0$ in $e^{0}\left([0, T] \times \mathbb{R}^{k}\right)$.

This allows us to obtain the first two results of convergence. To obtain the last one, we set $v^{\lambda}=\left(F^{\lambda}, G^{\lambda}, H^{\lambda}, L^{\lambda}\right) .\left\{v^{\lambda}\right\}$ is bounded in $\mathcal{C}^{0}\left([0, T] ; H^{s}\right),\left\{v_{t}^{\lambda}\right\}$ is bounded in $\mathcal{C}^{0}\left([0, T] ; H^{s-2}\right)$ (which yields the equicontinuity of $v^{\lambda}$ ). Therefore, using Ascoli-Arzela's theorem, we have the convergence of a subsequence in $\mathrm{e}^{0}\left([0, T] ; H_{\mathrm{loc}}^{s-2}\right)$.

Using interpolation and the boundedness in $\mathrm{e}^{\mathrm{o}}\left([0, T] ; H^{s}\right)$, we have the convergence of a subsequence in $\mathcal{C}^{0}\left([0, T] ; H_{\text {loc }}^{s-\varepsilon}\right) \forall \varepsilon>0$. Let $(\tilde{F}, \tilde{G}, \tilde{H}, \tilde{L})$ be the limit of this subsequence. 
Thanks to the equations governing $\left(F_{t}^{\lambda}, G_{t}^{\lambda}, H_{t}^{\lambda}, L_{t}^{\lambda}\right)$, we obtain the convergence in the sense of $\mathcal{C}^{1}\left([0, T] ; H_{\mathrm{loc}}^{s-2-\varepsilon}\right), \forall \varepsilon>0$. The equations pass to the limit

$$
\begin{gathered}
\tilde{F}_{t}-\frac{1}{2} B\left(\left(|\tilde{F}|^{2}+|\tilde{G}|^{2}\right) \tilde{G}\right)=-\Delta \tilde{G} \\
\tilde{G}_{t}+\frac{1}{2} B\left(\left(|\tilde{F}|^{2}+|\tilde{G}|^{2}\right) \tilde{F}\right)=\Delta \tilde{F} \\
\tilde{H}_{t}-A\left(\frac{1}{2}\left(|\tilde{F}|^{2}+|\tilde{G}|^{2}\right) \tilde{L}+\mathfrak{M}\left(\tilde{G}^{t} \tilde{G}\right) \tilde{L}+\mathfrak{M}\left(\tilde{G}^{t} \tilde{F}\right) \tilde{H}\right)=-\Delta \tilde{L}, \\
\tilde{L}_{t}+A\left(\frac{1}{2}\left(|\tilde{F}|^{2}+|\tilde{G}|^{2}\right) \tilde{H}+\mathfrak{K}\left(\tilde{F}^{t} \tilde{F}\right) \tilde{H}+\mathfrak{M}\left(\tilde{F}^{t} \tilde{G}\right) \tilde{L}\right)=\Delta \tilde{H}
\end{gathered}
$$

The regularity of the solutions enables us to return as before to the initial equations and we have

$$
i \tilde{E}_{t}+\Delta \tilde{E}=B\left(|\tilde{E}|^{2} \tilde{E}\right) \text {. }
$$

Remark. The different constants which are used in this section are said to depend only on $\delta$ (they, of course, also depend on the space dimension). We may be more precise and give additional information about how these constants depend on $\delta$ in order to estimate $T$.

In the proof of the lemma, we obtain in fact the following estimate

$$
\frac{\mathrm{d}}{\mathrm{d} t}\|u(t)\|_{0}^{2} \leq K_{I}\left(\delta+\delta^{3}\right)\|u(t)\|_{0}^{2}+2\|f\|_{0}\|u\|_{0} .
$$

During the estimation in large norm we have the following estimates

$$
\begin{gathered}
\left\|f_{1}\right\|_{0} \leq K_{1}\left(\delta+\delta^{3}\right) \mid U^{p+1} \|_{s}, \\
\frac{\mathrm{d}}{\mathrm{d} t}\left\|U^{p+1}(t)\right\|_{s}^{2} \leq K_{I J}\left(\delta+\delta^{3}\right)\left\|U^{p+1}(t)\right\|_{s}^{2}, \\
\left\|U^{p+1}(t)\right\|_{s}^{2} \leq \mathrm{e}^{K_{I I}\left(\delta+\delta^{3}\right) t} \varepsilon^{2}
\end{gathered}
$$

We also have the following estimate on $T$

$$
T \leq \frac{2}{K_{I I}\left(\delta+\delta^{3}\right)} \log \frac{\delta}{\varepsilon} .
$$

Studying the convergence in low norm, we have the following results

$$
\begin{gathered}
\left\|f_{2}\right\|_{0} \leq K_{2}\left(\delta+\delta^{3}\right) \mid V^{p-1} \|_{0}, \\
\beta=K_{I I I}\left(\delta+\delta^{3}\right), \\
\left\|V^{p}(t)\right\|_{0}^{2} \leq\left(\mathrm{e}^{\beta t}-1\right)\left\|V^{p-1}(t)\right\|_{0}^{2} .
\end{gathered}
$$

As we want $\beta T<\log 2$, this yields

$$
T<\frac{\log 2}{K_{I I}\left(\delta+\delta^{3}\right)} .
$$


Taking everything into account, we obtain that

$$
T \leq \frac{2}{K_{0}\left(\delta+\delta^{3}\right)} \log \frac{\delta}{\varepsilon} .
$$

In the rest of the section, no other restriction on $T$ is made.

We may think of some extensions of this study. For example, the same arguments as in [8] enable us to study the Cauchy problem for $\left(\phi_{0}, n_{0}, n_{1}\right) \in H^{m} \times H^{m-1} \times H^{m-2}$. There are opened problems for the Zakharov equation which are opened for this equation too. We may for example think of the global Cauchy problem in three dimensions.

Acknowledgements-I want to thank J. C. Saut for suggesting to me this subject and his encouragement, and T. Colin for helpful discussions.

\section{REFERENCES}

1. ZAKHAROV V. E., Collapse and self-focusing of Langmuir waves, in Basic Plasma Physics $I$ (Edited by A. A. GALEEV and R. N. SUDAN). North-Holland, Amsterdam (1984).

2. ZAKHAROV V. E., Collapse of Langmuir waves, Soviet Phys. JETP 35(5), 908-914 (1972).

3. COLIN T., Sur une équation de Schrödinger non linéaire et non locale intervenant en physique des plasmas, $C . r$. Acad. Sci. Paris, Serie I 314, 449-453 (1992).

4. ADDED H. \& ADDED S., Equations of Langmuir turbulence and nonlinear Schrödinger equation: smoothness and approximation, $J$. funct. Analysis 79, 183-210 (1988).

5. ADDED H. \& ADDED S., Existence globale de solutions fortes pour les équations de la turbulence de Langmuir en dimension 2. C. r. Acad. Sci. Paris, Serie I 299, 551-554 (1984).

6. SULEM C. \& SULEM P. L., Quelques résultats de régularité pour les équations de la turbulence de Langmuir, C. r. Acad. Sci. Paris 289, 173-176 (1979).

7. SCHOCHET S. H. \& WEINSTEIN M. I., The nonlinear Schrödinger limit of the Zakharov equations governing Langmuir turbulence, Communs math. Phys. 106, 569-580 (1986).

8. OZAWA T, \& TSUTSUMI Y., Existence and Smooting Effect of Solutions for the Zakharov Equations. (1990).

9. COLIN T., On a Nonlocal, Nonlinear Schrödinger Equation Occuring in Plasma Physics, Publication CMLA, ENS Cachan 9208 (1992).

10. GINIBRE J. \& VELO G., On a class of nonlinear Schrödinger equations I: the Cauchy problem, J. funct. Analysis 32, 1-32 (1979).

11. GINIBRE J. \& VELO G., Scattering theory in the energy space for a class of nonlinear Schrödinger equations, $J$. Math. pures appl. 64, 363-401 (1985).

12. STRICHARTZ R. S., Restrictions of Fourier transforms to quadratic surfaces and decay of solutions of wave equations, Duke math. J. 44, 705-714 (1977).

13. YAJIMA K., Existence of solutions for Schrödinger evolution equations, Communs math. Phys. 110, 415-426 (1987).

14. KATO T., Nonlinear Schrödinger equations, Lecture Notes for Physics, Vol. 345. Springer, Berlin 